\title{
Observation of shock-induced protein crystal damage during megahertz serial femtosecond crystallography
}

\author{
Marie L. Grünbein, ${ }^{1}$ Lutz Foucar, ${ }^{1}$ Alexander Gorel, ${ }^{1}$ Mario Hilpert, ${ }^{1}$ Marco Kloos, ${ }^{1, *}$ Karol Nass,,${ }^{1}$ Gabriela Nass Kovacs, ${ }^{1}$ \\ Christopher M. Roome, ${ }^{1}$ Robert L. Shoeman, ${ }^{1}$ Miriam Stricker, ${ }^{1}$ ॠ Sergio Carbajo, ${ }^{2}$ William Colocho, ${ }^{2}$ Sasha Gilevich, ${ }^{2}$ \\ Mark Hunter, ${ }^{2}$ Jim Lewandowski, ${ }^{2}$ Alberto Lutman, ${ }^{2}$ Jason E. Koglin, ${ }^{2,}{ }^{\S}$ Thomas J. Lane, ${ }^{2, \|}$ Tim van Driel, ${ }^{2}$ John Sheppard, ${ }^{2}$ \\ Sharon L. Vetter, ${ }^{2}$ James L. Turner, ${ }^{2}$ R. Bruce Doak, ${ }^{1}$ Thomas R. M. Barends, ${ }^{1}$ Sébastien Boutet, ${ }^{2}$ Andrew L. Aquila, ${ }^{2}$ \\ Franz-Josef Decker, ${ }^{2}$ Ilme Schlichting, ${ }^{1}$ and Claudiu A. Stan $\mathbb{1}^{3,4, \pi}$ \\ ${ }^{1}$ Max Planck Institute for Medical Research, Jahnstrasse 29, D-69120 Heidelberg, Germany \\ ${ }^{2}$ SLAC National Accelerator Laboratory, Menlo Park, California 94025, USA \\ ${ }^{3}$ Department of Physics, Rutgers University Newark, Newark, New Jersey 07102, USA \\ ${ }^{4}$ Stanford PULSE Institute, SLAC National Accelerator Laboratory, Menlo Park, California 94025, USA
}

(Received 13 October 2020; accepted 21 December 2020; published 15 January 2021)

\begin{abstract}
Shock waves launched by x-ray pulses in sample-carrying liquid jets may affect protein crystallography data collected at $\mathrm{MHz}$ repetition rate $\mathrm{x}$-ray free-electron laser (XFEL) facilities, by damaging the crystals before they are probed. We investigated the shock damage in lysozyme microcrystals using a double-pulse operation mode at a low repetition rate $\mathrm{x}$-ray laser facility. The double-pulse mode generated shock waves with pressures that covered and exceeded the shock pressures expected at $\mathrm{MHz}$ pulse rate experiments at the European XFEL (EuXFEL) $\mathrm{x}$-ray laser. The quality of the x-ray diffraction data from the crystals was degraded after the shock passed. A decrease in the number of peaks and in the resolution occurred above an estimated shock pressure threshold on the order of tens of MPa. Based on the scaling of the shock pressure with the sample injection parameters and the pulse rates, this threshold was not reached in initial EuXFEL experiments performed at pulse rates of $1.1 \mathrm{MHz}$ but may be exceeded at the maximum pulse rate of $4.5 \mathrm{MHz}$. The observation of shock damage in lysozyme crystals indicates how experiments can be designed to rapidly detect, and eventually avoid, shock damage in other crystals. Our analysis of shock pressures in liquid jets can also be used to estimate the effect of the shocks in other types of experiments at MHz repetition rate XFELs.
\end{abstract}

DOI: 10.1103/PhysRevResearch.3.013046

\section{INTRODUCTION}

X-ray free-electron lasers (XFELs) are the most advanced type of X-ray generation facilities $[1,2]$, and their capacities are developing rapidly to satisfy demand for an increasing number of applications. The number of photons in the pulses produced by XFELs is extremely high and even weakly

\footnotetext{
*Current address: European XFEL GmbH, Holzkoppel 4, 22869 Schenefeld, Germany.

${ }^{\dagger}$ Current address: Paul Scherrer Institut, Forschungsstrasse 111, 5232 Villigen, Switzerland.

*Current address: Department of Statistics, University of Oxford, 24-29 Giles St., Oxford OX1 3LB, United Kingdom.

${ }^{\S}$ Current address: Los Alamos National Laboratory, P.O. Box 1663, Los Alamos, NM 87545, USA.

"Current address: Center for Free-Electron Laser Science, DESY, Notkestrasse 85, 22607 Hamburg, Germany.

II claudiu.stan@ rutgers.edu
}

Published by the American Physical Society under the terms of the Creative Commons Attribution 4.0 International license. Further distribution of this work must maintain attribution to the author(s) and the published article's title, journal citation, and DOI. scattering microscopic objects can be investigated using single pulses $[3,4]$, while the femtosecond duration of the pulses allows the investigation of ultrafast processes such as chemical reactions at the molecular timescale [5-7]. Aside from femtosecond processes, XFELs can acquire unique information from systems whose dynamics are stochastic or very difficult to induce, at nanosecond [8-11] or even longer timescales $[12,13]$, because XFELs can capture shortlived intermediate states using single-shot measurements. The applications of XFELs include the ability to perform measurements on samples that would be damaged before data are acquired during a continuous exposure to $\mathrm{x}$ rays because XFEL pulses can outrun radiation damage [14]. This principle enabled one of the most successful applications of XFELs yet, serial femtosecond crystallography (SFX) [3,15].

A decade of research at the first-generation XFELs, which have pulse rates between 10 and $120 \mathrm{~Hz}$, led to many discoveries in physics, chemistry, biology, and material science [16]. An increase in the amount and diversity of the research is expected from the second-generation XFELs, which use superconducting linear electron accelerators and produce XFEL pulses at rates on the order of $1 \mathrm{MHz}$. The first operational second-generation XFEL is the European XFEL (EuXFEL) in Germany [17]. This facility is designed to generate up to 27000 pulses per second, delivered in 10 trains per sec- 
ond with a $4.5-\mathrm{MHz}$ repetition rate within each train. This is a more than 200-fold increase in the pulse rate over the first-generation XFELs. Two other XFELs under construction, LCLS-II in Menlo Park, California [18], and SHINE in Shanghai, China [19], will improve the average pulse rate further by operating continuously at pulse rates of $1 \mathrm{MHz}$. Second-generation XFELs can therefore reduce the time needed for data acquisition, which makes XFELs available to more scientists because a larger number of experiments can be scheduled.

Determination of the three-dimensional structure of a molecule by SFX requires thousands of diffraction images. Second-generation XFELs can speed up SFX data acquisition by one to two orders of magnitude and can greatly improve the number of time points in SFX experiments studying dynamics in which multiple intermediate structures are determined during a biochemical process. However, taking full advantage of the higher pulse rates depends on being able to deliver fresh samples at the same rate. Out of the existing sample delivery methods [20], only high-speed liquid microjets produced by gas dynamic virtual nozzles (GDVN) [21,22] can deliver crystals for SFX at sufficiently high speeds to use the full pulse rates at EuXFEL.

The liquid microjets need to clear not only the region irradiated by the previous pulse, but also a larger region damaged by ablation. The high-intensity XFEL pulses vaporize a segment of the jet at the point of irradiation and generate a gap in the jet [23]. The gap translates with the jet and a continuous jet will eventually be reestablished at the interaction point, but subsequent XFEL measurements are not possible until the gap is cleared. Models for the gap formation [23] predict that a jet velocity of $\approx 100 \mathrm{~m} / \mathrm{s}$ is required to clear the gap between pulses at 4.5 MHz; such jets have been already developed [24].

The XFEL pulse also launches a shock wave in the jet medium [23], which propagates along the jet and may damage the samples carried by the jet, such as microcrystals in SFX. Measurements in jets larger than the ones used for SFX showed that the initial pressure of the shock is on the order of $1 \mathrm{GPa}$. The shock pressure decreases rapidly in amplitude as it travels along the jet and generates secondary trailing shocks [25]. The initial (leading) shock and the trailing shocks travel together in the jet, forming a "shock train" [23]. Depending on their peak pressure, these shocks may lead to damage in crystals because their pressure can exceed the mechanical strength of certain protein crystals; for example, the estimated compression strength of tetragonal lysozyme crystals is $\approx 10 \mathrm{MPa}$ [25]. The shocks may also change the structure of protein molecules [26].

Since the peak pressure of the leading shock decreases as the train travels in the jet, the risk of sample damage increases as the distance between regions hit by consecutive pulses decreases. Since this distance becomes smaller as the pulse rate increases, the highest risk of sample damage at second-generation XFELs will be encountered during 4.5-MHz operation at the EuXFEL.

Several initial SFX studies conducted at EuXFEL investigated the existence of shock damage [27-29], searching for a degradation of the diffraction resolution in data sets recorded at pulse rates of $1.1 \mathrm{MHz}$. Although none of these studies reported shock-induced damage, they do not imply that dam- age will not occur at the maximum pulse rate of EuXFEL, $4.5 \mathrm{MHz}$, because the risk of shock damage increases with the pulse rate. As the EuXFEL is approaching routine operation at $4.5 \mathrm{MHz}$, the main questions about shock damage are (i) whether it will occur, (ii) what are the most efficient ways to identify it (preferably at the beginning of data acquisition), and (iii) whether it can be predicted for a given experiment.

We performed two time-resolved X-ray pump X-ray probe SFX experiments at the Linac Coherent Light Source (LCLS) [1] using closely spaced pairs of pulses (separated by 8.4 and $122.5 \mathrm{~ns}$ ) that allowed us to investigate the effect of the shock waves under conditions that are relevant to a $4.5-\mathrm{MHz}$ experiment at the EuXFEL. Comparison of diffraction data from shocked crystals and not-shocked crystals revealed shock-induced damage, as a significant degradation of diffraction quality. At the same XFEL pulse energies the degradation was more severe in the 8.4-ns experiment than in the 122.5-ns experiment because the crystals were subjected to higher shock pressures. The degradation was similar in both experiments when measured as a function of shock pressure. We modeled the shock pressure based on recent experimental studies of shock trains in liquid jets [25,30]. Using this model, we also estimated the shock pressures expected in future SFX experiments at EuXFEL, and we predict that shock damage may occur for certain sample injection parameters and XFEL settings.

\section{EXPERIMENTAL DESIGN}

The experiment was designed to determine if damage occurs in protein crystals subjected to shock waves similar to those expected in a $4.5-\mathrm{MHz}$ experiment at EuXFEL. Although the EuXFEL experiment will involve sequences of up to 2700 pulses at $4.5 \mathrm{MHz}$ (or $222 \mathrm{~ns}$ between consecutive pulses), sequences of only two pulses (pulse pairs) are sufficient to investigate the existence and the signatures of shock damage because the second pulse probes crystals exposed to the shock generated by the first pulse.

To generate the pulse pairs, LCLS was operated in a "twobunch" mode that produces nanosecond-spaced pairs of pulses at a rate of 120 pairs per second [31-33]. A detailed description of the "two-bunch" mode used in our experiments, and of the diagnostics we implemented, is given in Appendix A. In this mode, the pulses can be separated by up to $\approx 200 \mathrm{~ns}$ within a pair, and each of the pulses can have a total energy of up to $\approx 1 \mathrm{~mJ}$. While these parameters are close to the parameters of two consecutive $4.5-\mathrm{MHz}$ pulses at EuXFEL, additional features were needed at LCLS to record and diagnose data from each pulse in the pair, and to compensate for the fact that the in-pair delays we used were shorter than 222 ns.

We performed the two-bunch experiments in the microfocus chamber of the Coherent X-ray Imaging (CXI) end station [34], the first at a pulse delay of $8.4 \mathrm{~ns}$, and the second at $122.5 \mathrm{~ns}$. The setup of the 122.5-ns experiment is illustrated in Fig. 1, and the 8.4-ns setup was identical except for the pulse diagnostics setup (see Appendix A). Hen egg-white lysozyme (HEWL) protein microcrystals were injected into the $\mathrm{x}$-ray interaction region in a GDVN liquid microjet [22] with an average diameter of $\approx 5 \mu \mathrm{m}$ and $\approx 50 \mathrm{~m} / \mathrm{s}$ velocity. The first pulse induced a jet explosion and launched shocks 

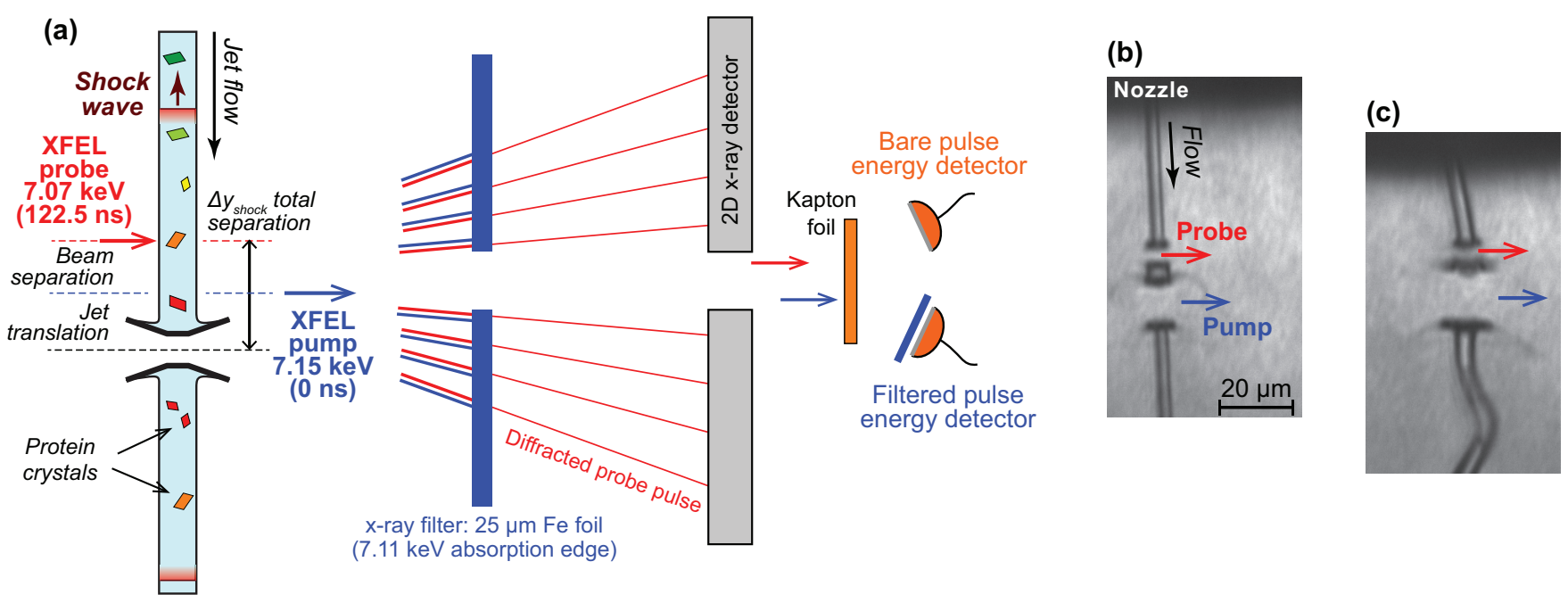

FIG. 1. (a) Experimental setup: two XFEL pulses hit in rapid succession a liquid jet carrying protein microcrystals. The first pulse (pump) launches a shock that passes through the crystals probed by the second pulse (probe) 8.4 or $122.5 \mathrm{~ns}$ later. The photon energies of the pulses are different and chosen such that only the x-ray diffraction from the probe pulse is measured. The total energies of the pulses are measured by fast photodiodes for further data processing. (b), (c) Images of the jets for 122.5-ns pulse separation, showing the generation of jet gaps by both pulses, in pure water jets (b) and in jets carrying crystals (c).

along the jet, and the second pulse probed crystals that had been subjected to the shocks. To ensure that the second pulse was passing through the liquid jet instead of the gap created by the explosion, the two pulses were displaced vertically by $\Delta y_{\text {pulse }}=5 \mu \mathrm{m}$, such that the interaction region with the probe pulse was upstream of the pump pulse (i.e., towards the injection nozzle).

The vertical separation of the pulses also increased the separation $\Delta y_{\text {shock }}$ between the locations where the pulses hit, in the reference frame of the jet. The probed crystals were thus a distance $\Delta y_{\text {shock }}$ from the place where the pump pulse intersected the jet and the shock was generated. When the probed crystals are subjected to transient pressures as the shock train passes, damage is expected to occur only if the maximum pressure in the shock train exceeds the pressure threshold for damage. The maximum pressure at the sample is thus expected to be the dominant factor for inducing damage, and it decays as $\Delta y_{\text {shock }}$ increases [25]. Therefore, for comparing our study with a $\mathrm{MHz}$ repetition rate experiment, $\Delta y_{\text {shock }}$ is a better parameter to use than the temporal separation of the two pulses $\Delta t_{\text {pair }}$. As shown in Fig. 1, $\Delta y_{\text {shock }}$ is equal to the sum of $\Delta y_{\text {pulse }}$ and of the jet translation between pulses $\Delta y_{\text {jet }}=$ $v_{\text {jet }} \Delta t_{\text {pair }}$. In a standard SFX experiment, the pulses arrive at the same point in space and $\Delta y_{\text {shock }}=\Delta y_{\text {jet }}=v_{\text {jet }} \Delta t_{\mathrm{XFEL}}=$ $v_{\text {jet }} / f_{\text {XFEL }}$, where $\Delta t_{\mathrm{XFEL}}$ is the temporal separation between pulses and $f_{\mathrm{XFEL}}$ is the frequency of the pulses. Therefore, our experiments probed conditions that are equivalent to running a standard SFX experiment at an equivalent pulse frequency $f_{\text {XFEL }}=v_{\text {jet }} / \Delta y_{\text {shock }}$, which was equal to 9.2 and $4.5 \mathrm{MHz}$, respectively, for the 8.4- and 122.5-ns experiments.

The standard x-ray detectors and diagnostics at LCLS cannot resolve data from XFEL pulses that are $\approx 100 \mathrm{~ns}$ apart. To separate the diffraction patterns, we used a two-color scheme for the x-ray pump x-ray probe experiment $[35,36]$. The photon energies of the first ("pump") and second ("probe") pulses were set $\approx 40 \mathrm{eV}$ above and below the iron $\mathrm{x}$-ray absorption $\mathrm{K}$ edge ( $7.11 \mathrm{keV})$, respectively. A $25-\mu \mathrm{m}$-thick iron foil, placed between the samples and the Cornell-SLAC Pixel Array Detector (CSPAD), absorbed selectively the diffracted photons from the pump pulse, thus the diffraction pattern recorded by the detector was due to the probe pulse only (Fig. 1). Diffraction data from crystals not affected by shocks were recorded in separate measurements during which the pump pulse was suppressed.

The energies of the pump and probe pulses were measured for each shot by fast photodiodes monitoring the diffuse scattered $\mathrm{x}$ rays from materials placed after the sample, such as the Kapton foil shown in Fig. 1 (see Appendix A for details). To verify that the pulses had the proper photon energies above and below the absorption edge, in the 8.4-ns experiment, the combined spectrum from both pulses was monitored and recorded using an inline single-shot spectrometer [37]. In the 122.5-ns experiment, a second photodiode was placed behind a $25-\mu \mathrm{m}$-thick iron foil (see Fig. 1) to verify for each shot that the pump pulse was almost completely absorbed by the foil and thus did not contribute to the recorded diffraction patterns. The second photodiode could detect below-edge pump photons if they had more than $\approx 2 \%$ of the average energy of the probe pulse.

The interaction between the jet and the pulses was independently diagnosed using femtosecond optical imaging [23]. Jet images were recorded approximately when the probe pulse arrived, to confirm that the pump pulse induced a jet explosion, and therefore hit the jet. The imaging setup was also used to determine the jet velocity and the vertical separation of the pulses (see Appendix B for details on the sample jets). We note that the shock waves could not be imaged reliably in the GDVN jets. In GDVN jets with $\approx 5 \mu \mathrm{m}$ diameter, the optical path difference induced by the shock compression is smaller than for the shocks observed in (14-30)- $\mu$ m-diameter jets [25], and the shocked region did not deflect the imaging light enough make the shocks visible.

We chose to conduct our measurements using micron-sized crystals of hen egg-white lysozyme because they are the most 

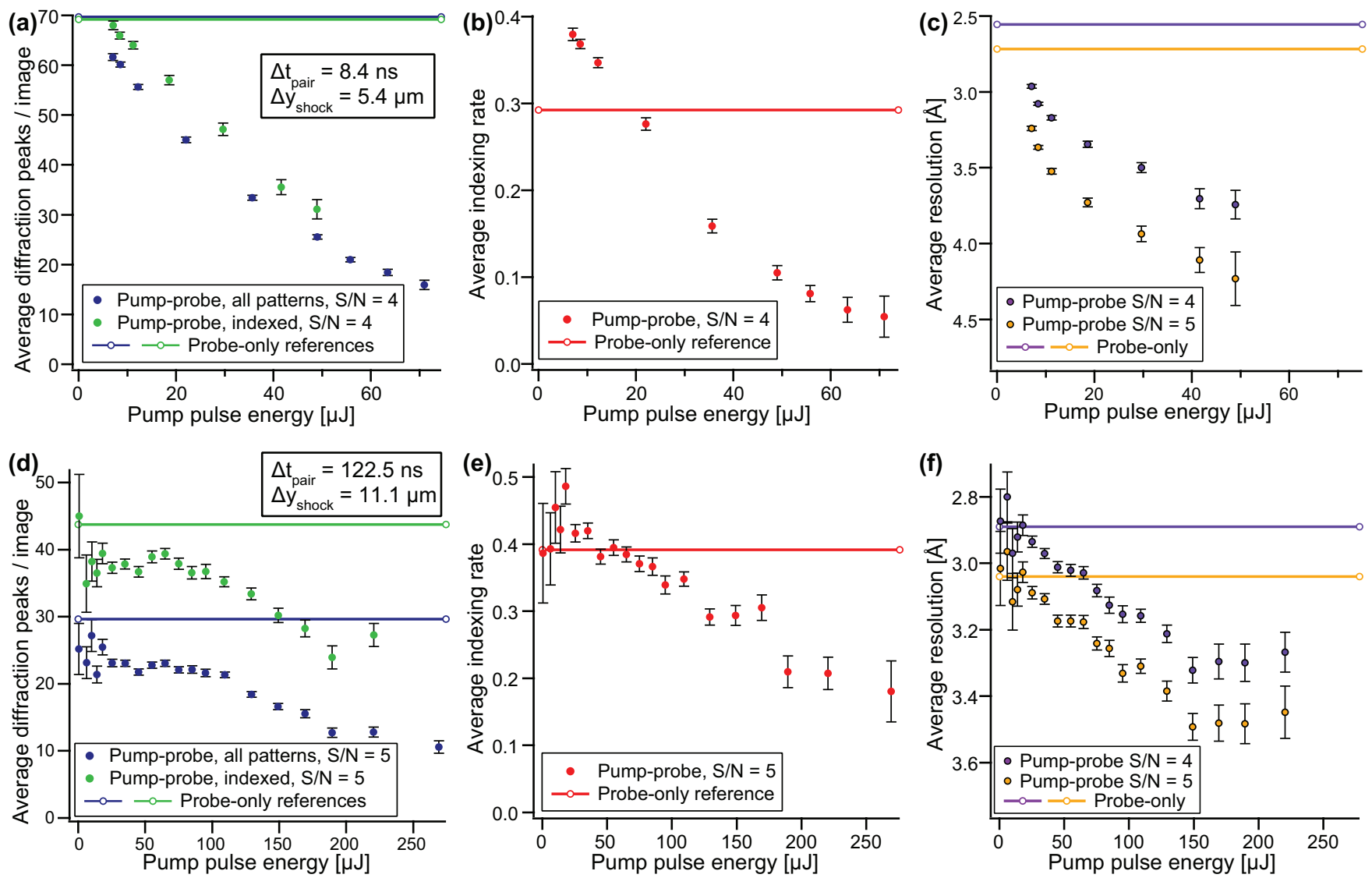

FIG. 2. Degradation of x-ray diffraction patterns as a function of the pump-pulse energy in (a)-(c) the 8.4-ns experiment and in (d)-(f) the 122.5-ns experiment.

common test sample used in SFX experiments, including the initial studies at EuXFEL [27,28]. Also, lysozyme crystals offer good opportunities to quantify and interpret shock damage because their pressure-dependent behavior, including structural transformations and damage, was investigated at high static pressures [38,39] and under mechanical compression $[40,41]$. The preparation of the crystals and their delivery into the $\mathrm{x}$-ray beam via liquid jet injection are described in Appendix B.

\section{RESULTS}

\section{A. Observation of shock-induced crystal damage}

The first experiments were conducted using pulses separated by $8.4 \mathrm{~ns}$ temporally and $\approx 5 \mu \mathrm{m}$ vertically. In the pump-probe runs, we monitored in real time the size of the gap made by the pump pulse when the probe pulse arrived (i.e., at a 8.4-ns delay after the pump), the energy of the pump pulse as measured by the fast photodiodes, the diffraction images recorded by the CSPAD detector, and the "hit rate" as reported by online analysis of the diffraction images in CASS $[42,43]$. The hit rate was defined as the fraction of diffraction images containing at least 10 diffraction peaks detected by CASS. Compared to calibration runs with single pulses, pump-probe operation led to a decrease in the hit rate, which became more pronounced as the pump-pulse energy increased. This decrease in hit rate occurred even if the pump-pulse energy was so low that it did not produce gaps in the jet at $8.4 \mathrm{~ns}$, therefore, it could not be caused by the probe pulse passing through the gap (thus failing to intercept crystals). At higher pump-pulse energies, a gap formed and the diffraction images displayed in real time had fewer diffraction peaks, indicating a clear degradation of the diffraction quality.

To quantitatively analyze the shock-induced damage, we investigated the dependence of the number of diffraction peaks in all hits, of the indexing rate (fraction of hits that can be indexed), of the number of peaks in indexed hits, and of the diffraction resolution, on the pump-pulse energy. All these variables were calculated using peaks that exceeded fixed signal-to-noise ratio $(S / N)$ thresholds, except the indexing rate where all the peaks were used. Before performing the analysis, (i) we eliminated XFEL shots in which the jet was not hit by both pump and probe, (ii) we binned the data by the pump-pulse energy, (iii) we selected the data such that the distribution of the probe-pulse energies were the same in the probe-only data and each of the bins in the pump-probe data, and (iv) for the 8.4-ns experiment we improved the accuracy of the pump-pulse energy measurements through a deconvolution-based analysis. These analysis steps, described in Appendix $\mathrm{C}$, account for the variability of XFEL pulses, and for a small signal-to-noise ratio of the fast diode energy detectors in the 8.4-ns experiment.

The dependencies of the average number of diffraction peaks per diffraction image, of the average indexing rate, and of the average resolution of the diffraction patterns as a function of the pump-pulse energy are shown in Fig. 2 for 
both the 8.4- and the 122.5-ns experiments. Although the hit rate also degraded, we did not perform a quantitative analysis because the crystal concentration in the jet was unstable in part of the 8.4-ns data due to clogging, which precluded the determination of a constant reference value. The pump energies displayed in Fig. 2 represent the value of the pulse energy at the XFEL source because the fast diode energy detectors were calibrated against the LCLS gas energy detector. The energy at the sample will be lower than at the source due to the beamline transmission loss, which was not measured for our experiments but is estimated to be around $50 \%$.

The degradation of diffraction patterns was observed at approximately one order of magnitude lower pump energies in the 8.4-ns experiment [Figs. 2(a)-2(c)] than in the 122.5-ns experiment [Figs. 2(d)-2(f)]. This difference is due to the longer shock travel distance, and correspondingly to a larger decay of the shock pressure, in the 122.5-ns experiment.

The average resolution was more sensitive to the pulse energy than the number of peaks: in the 122.5-ns data the resolution [Fig. 2(f)] degraded significantly above $\approx 30 \mu \mathrm{J}$, but the number of peaks [Fig. 2(d)] remained approximately constant up to $\approx 100 \mu \mathrm{J}$. This difference in sensitivity is most likely due to the relatively larger degradation of peak intensities at high resolution (see Supplemental Material [44]).

The data shown in Fig. 2 focus on basic diffraction parameters and are based on analyzing Bragg peaks with high signal-to-noise ratios. This analysis is effective for determining shock-induced degradation from relatively small data sets and has a high sensitivity to the pump-pulse energy, but the parameter values are not identical to the ones obtained when the data are processed for structural determination. The Supplemental Material [44] discusses these differences and other features of the data.

In addition to the degradation of the basic measures of diffraction quality (Fig. 2), degradation was also observable in the statistics of the integrated diffraction intensities when comparing probe-only with pump-probe data (see Supplemental Material [44]). For shocked crystals, the mean integrated intensities as well as signal-to-noise ratio of the integrated intensities exhibited a larger relative degradation at high resolution. These features as well as the increase of the Wilson B-factor in the shocked crystal data indicate a higher overall disorder of the crystal lattice, caused by stochastic displacements of the molecules or of larger entities such as mosaic blocks.

In principle, the degradation of the diffraction data could be caused by radiation damage from photons in the outer regions of the pump beam. However, when evaluating the electron densities, the pump-probe data did not display any signatures of radiation damage (see Appendix C), leaving shock damage as the only known cause of degradation. We also note that since the shocks last only a few nanoseconds, the crystals were probed after the shocks passed and the pressure returned to its initial value, therefore, the damage persisted up to at least $122.5 \mathrm{~ns}$. A residual pressure at the sample is possible in the 8.4-ns experiment, where based on the geometric and temporal scaling of the shock train patterns observed in larger jets [25], the crystals would overlap with a hypothetical fourth shock in the train when probed. In larger diameter jets, a fourth shock did not develop yet at time delays scaled to the 8.4-ns experiment, therefore, we expect the residual pressure to be much smaller than the maximum pressure experienced by the sample. The unit cell and the structure of the protein derived from the pump-only and pump-probe data did not change (see Appendix C) in either of the experiments, which means that any residual pressures were too small to induce a detectable compression of the crystals.

\section{B. Estimation of shock pressure thresholds for damage}

Shock waves generate a rapid compression along the direction of shock travel. Since the protein crystals are solid materials, they can be compressed elastically to a certain extent, but will be damaged if the applied pressure exceeds a damage threshold. Therefore, we investigated if we could determine empirically a shock pressure threshold above which the damage becomes observable in the diffraction data. To determine this threshold, the shock pressures were estimated from the pump-pulse energy.

No experimental measurements have been reported yet on the shock amplitude and decay in $\approx 5$ - $\mu \mathrm{m}$-diameter liquid jets. Nevertheless, measurements of XFEL-induced shock pressure in 14- and $20-\mu \mathrm{m}$ jets [25], and recent experiments on shocks produced using optical lasers in jets with diameters up to $70 \mu \mathrm{m}$ [30], indicate that the decay of shocks for propagation distances up to 2-3 jet diameters is exponential, with a decay rate inversely proportional to the jet diameter. The experiments with optical lasers also reported that the shock trains reach an approximately constant pressure of $\approx 40 \mathrm{MPa}$. An additional shock decay mechanism, modeled as the ultrasonic attenuation of a shock train, was proposed by Blaj et al. [25] for propagation distances larger than 2-3 jet diameters. The ultrasonic attenuation also becomes stronger as jet the diameter decreases.

We developed a mathematical model of the shock wave decay that combines the experimental observations with the ultrasonic decay hypothesis by multiplying the equation proposed by Ursescu et al., which accounts the rapid decay down to the $\approx 40 \mathrm{MPa}$ constant pressure [30], with the ultrasonic decay proposed by Blaj et al. [25]:

$$
\begin{gathered}
P(x)=\left\{P_{\text {long }}+\left[P(R)-P_{\text {long }}\right] \times 10^{-(x-R) /(3 R)}\right\} e^{-\alpha_{f}(x-R),} \\
\alpha_{f}=2.53 \times 10^{-14} f^{2}=5.69 \times 10^{-8} R^{-2}
\end{gathered}
$$

where $x$ is the propagation distance, $R$ is the jet radius, $P(x)$ is the pressure of the leading shock, $P_{\text {long }}=40 \mathrm{MPa}$ is the long-lived shock pressure observed in $30-\mu \mathrm{m}$ diameter jets, $\alpha_{f}$ is the ultrasonic attenuation coefficient in water, and $f$ is the shock train frequency. For the calculation of $\alpha_{f}$ we used Pinkerton's data [45] and a center frequency $f=c_{0} / R$ [25], where $c_{0}$ is the speed of sound in water.

The shock pressure at $x=R, P(R)$, was calculated by scaling the pressure data reported by Blaj et al. [25] with the energy absorption coefficient of $\mathrm{x}$ rays and with $R$. We fit the data from Ref. [25] to determine a pressure coefficient of $648 \mathrm{MPa} / \mathrm{mJ}$ at a distance of $10 \mu \mathrm{m}$ for $9.53-\mathrm{keV}$ x rays. At different photon energies, we scaled this coefficient by the linear energy absorption coefficient in water. Here, all pressure calculations were done for jets of water, whose $\mathrm{x}$-ray 
absorption is similar to that of solutions containing organic crystallization precipitants such as polyethylene glycol. Jets containing inorganic salt precipitants (e.g., $\mathrm{NaCl}, \mathrm{NH}_{4} \mathrm{SO}_{4}$ ) absorb hard $\mathrm{x}$ rays more strongly (up to a factor of 2 for a $\approx(2-3)$-M salt solution), resulting in correspondingly stronger shocks. We also assumed that until the shock contacts fully the jet surface, its pressure is inversely proportional with the propagation distance, a simple model supported by measurements of XFEL-induced shocks in water droplets [46]. For $7.15-\mathrm{keV}$ x rays at $R=2.5 \mu \mathrm{m}$, we obtained a proportionality coefficient of $6.2 \mathrm{GPa} / \mathrm{mJ}$ between the energy of the pump pulse at source and $P(R)$.

Equation (1) applies when $P(R)$ is larger than $P_{\text {long }}$, which is the case for a typical SFX experiment. In our experiments, for the lowest pulse energy bins, $P(R)<P_{\text {long. }}$. In this case we assumed that the main decay mechanism is the ultrasonic attenuation, and we calculated the shock pressure with a simplified formula

$$
P(x)=P(R) e^{-\alpha_{f}(x-R)} .
$$

The predictions given by Eqs. (1) and (3) are based on experiments that report a consistent qualitative and quantitative behavior of the ablation-driven shock trains in liquid jets, and reproduce the results of these experiments (see Appendix D). The equations are based on extrapolations of data recorded in jets with diameters larger than the ones used in SFX, and these extrapolations limit the predictive accuracy of the equations. Nevertheless, until direct measurements or numerical simulations of shocks in SFX jets become available, these equations can be used to compare the shock pressures in different experiments.

The average number of all diffraction peaks per diffraction image and the average diffraction resolution of indexed diffraction peaks with high signal-to-noise ratio are shown in Fig. 3 as a function of the estimated shock pressure. Among the basic diffraction quality measures we investigated, the number of all peaks is the fastest to evaluate during a beam time because it does not involve indexing, while the resolution of indexed peaks with high signal-to-noise ratios was the most sensitive to damage. When displayed against the shock pressure, the damage in the 8.4- and the 122.5-ns experiments becomes similar. Since the experiments differ in the time elapsed between the arrival of the shock at the crystal and the probe pulse, the similar dependence on the peak shock pressure indicates that the degree of damage is either independent of, or has a weak dependence on, how much time has passed after the crystal was shocked, up to at least $\sim 100 \mathrm{~ns}$. Therefore, the shock pressure can be used to quantify the shock damage in MHz SFX experiments even if the time interval between pulses varies.

In Fig. 3, the dependence of the diffraction parameters of lysozyme microcrystals on the shock amplitude shows that the damage occurred only above certain pressure thresholds. The 122.5-ns experiment provided sufficient data at low shock pressures to measure damage thresholds, which we defined as the shock pressure above which the degradation of a diffraction parameter exceeds by one standard deviation its value in the lowest pressure bin. The precise location of the thresholds will depend on the signal-to-noise data cutoffs; the dependence of the integrated intensity on resolution
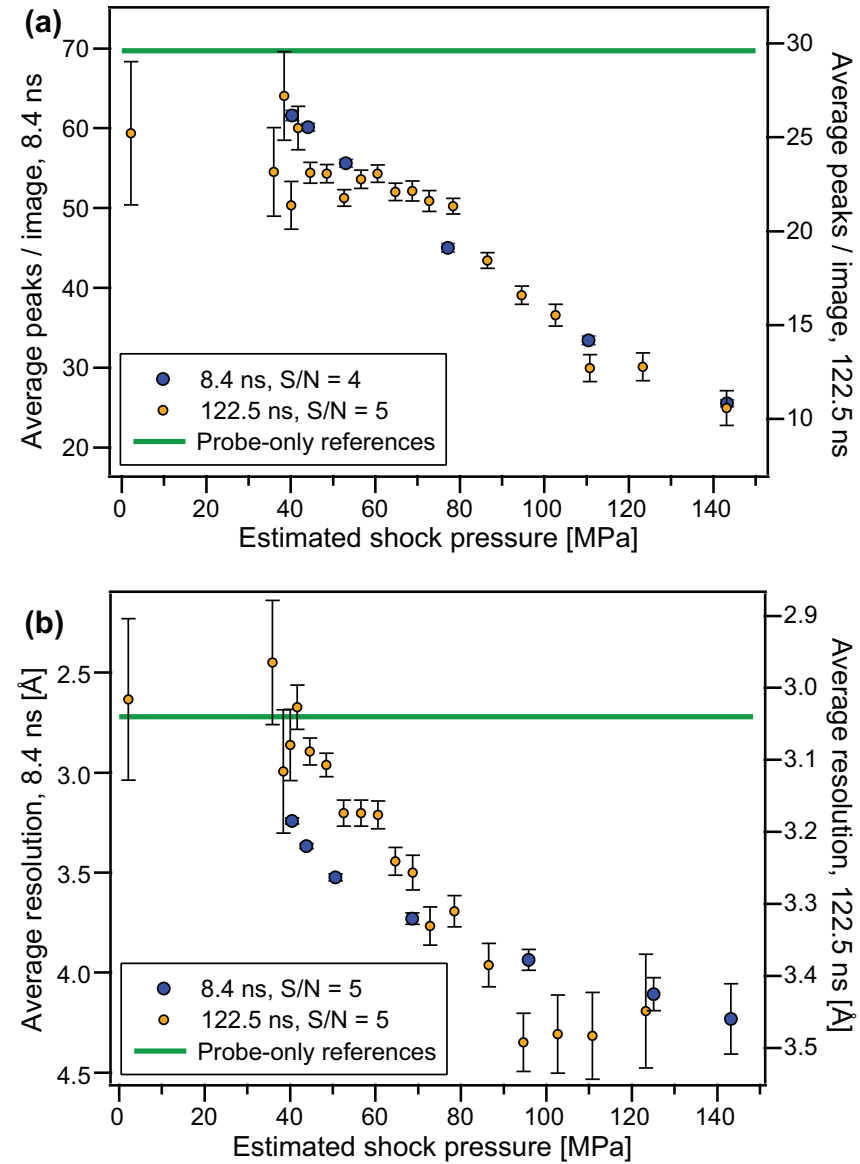

FIG. 3. Degradation of (a) the average number of peaks per diffraction image detected by CRYSTFEL, and (b) the average diffraction resolution, from micron-sized lysozyme crystals, as a function of the estimated shock pressure. The left and right axes are scaled such that the probe-only references at 8.4 and 122.5 ns overlap.

(see Supplemental Material [44]) suggests that the measured thresholds will be lower if the signal-to-noise cutoff is lower. Nevertheless, the present thresholds allow the comparison of different experiments and different samples.

The lowest pressure for a significant decrease in the peak numbers was $\approx 80 \mathrm{MPa}$, and the lowest pressure for a significant decrease in resolution was $\approx 45 \mathrm{MPa}$. Since the resolution of indexed diffraction peaks was more sensitive to shock damage, the 122.5-ns measurements indicate a damage threshold of $\approx 45 \mathrm{MPa}$. In the 8.4-ns experiment we could not make measurements at shock pressures below $\approx 40 \mathrm{MPa}$ due to a relatively higher noise of the pulse energy measurements; the onset of damage can only be estimated, with less accuracy than in the 122.5-ns experiment, at either $\approx 30 \mathrm{MPa}$ (as the pressure where the extrapolation of data intercepts the reference value) or $\approx 45 \mathrm{MPa}$ (as the first data point displaying a significant degradation relative to points at lower pressures). Due to the high noise of pulse energy measurements in the 8.4ns experiment (see Appendix C), the difference between the damage thresholds in the 8.4- and the 122.5-ns experiments is not statistically significant. Overall, the shock damage threshold in lysozyme was observed to range from 30 to $45 \mathrm{MPa}$, for data sets of less than 10000 diffraction images processed with high signal-to-noise cutoffs. These values are several 
TABLE I. Estimated and predicted shock wave pressures for MHz repetition rate SFX experiments. All experiments probed HEWL crystals.

\begin{tabular}{|c|c|c|c|c|c|c|c|}
\hline Description & $\begin{array}{l}\text { Pulse } \\
\text { rate } \\
(\mathrm{MHz})\end{array}$ & $\begin{array}{c}\text { Photon } \\
\text { energy } \\
(\mathrm{keV})\end{array}$ & $\begin{array}{l}\text { Pulse energy } \\
\text { at sample } \\
(\mathrm{mJ})\end{array}$ & $\begin{array}{c}\text { Average } \\
\text { jet diameter }{ }^{\mathrm{a}} \\
(\mu \mathrm{m})\end{array}$ & $\begin{array}{c}\text { Jet } \\
\text { velocity } \\
(\mathrm{m} / \mathrm{s})\end{array}$ & $\begin{array}{c}\text { Shock } \\
\text { pressure }^{\mathrm{b}} \\
(\mathrm{MPa})\end{array}$ & $\begin{array}{c}\text { Damage } \\
\text { observed? }\end{array}$ \\
\hline This work, ${ }^{\mathrm{c}} 122.5 \mathrm{~ns}$ & 4.5 & 7.15 & $>0.013$ & 5 & 50 & $>45$ & Yes \\
\hline Grünbein et al. ${ }^{\mathrm{d}}$ [27] & 1.1 & 7.47 & $0.6-1.1$ & 4 & 50 & $<22^{\mathrm{e}}$ & No \\
\hline Wiedorn et al. [28] & 1.1 & 9.3 & 0.29 & 3 & 100 & $<4.4^{\mathrm{e}}$ & No \\
\hline Yefanov et al. [29] & 1.1 & 9.3 & 0.8 & $2-3$ & 100 & $0.3-4.4$ & No \\
\hline Full rate with margins & 4.5 & 9.3 & 1 & 3 & 100 & 24 & \\
\hline Half-rate with margins & 2.25 & 9.3 & 1 & 3 & 100 & 14 & \\
\hline Full rate at gap clearing limit & 4.5 & 7.1 & 2 & 4 & 90 & 55 & \\
\hline Half-rate at gap clearing limit & 2.25 & 7.1 & 2 & 4 & 90 & 23 & \\
\hline
\end{tabular}

$\overline{\bar{a}}$ The average jet diameters were determined using the measured sample flow rate and jet velocity, except for this work where they were measured from jet images.

${ }^{\mathrm{b}}$ The calculated pressures assume jets that have the same $\mathrm{x}$-ray absorption as water.

${ }^{\mathrm{c}}$ The listed XFEL frequency is the equivalent pulse frequency, and the pulse energy at sample assumes $50 \%$ beam line transmission.

${ }^{\mathrm{d}}$ Pulse energies at the sample were calculated from the upper limit of the quoted energies at the source, multiplied by a beam line transmission factor of $70 \%$.

${ }^{\mathrm{e}}$ The first reported SFX experiments at EuXFEL used 15- $\mu \mathrm{m}$ FWHM beams, larger than the jet diameters, therefore, the model will overestimate the shock pressure.

times larger than the uniaxial compression strength of $10 \mathrm{MPa}$ proposed by Blaj et al. [25] for lysozyme crystal damage. Further work is needed to improve the measurement accuracy of the damage thresholds in SFX, and to explore how they relate to the uniaxial compression strength of protein crystals.

\section{DISCUSSION}

\section{A. Implications for MHz XFEL experiments}

Although the equivalent pulse rate in the 122.5-ns experiment $(4.5 \mathrm{MHz})$ matches the maximum EuXFEL pulse rate, the relation between the pulse energy and the shock pressure was different from that expected in EuXFEL experiments, which are likely to use thinner and faster jets to clear a larger jet gap at $222 \mathrm{~ns}$. A different jet diameter and velocity will lead to a different degree of shock pressure attenuation between pulses, and the XFEL pulses will have different parameters from the ones we used. Therefore, it is more accurate to compare the experiments in terms of the shock pressure at the samples. To evaluate the risk of shock damage we used Eqs. (1) and (3) to estimate the shock pressures for several 1.1-MHz SFX experiments reported from EuXFEL and compared them in Table I to the corresponding pressures in our experiments, and to pressures predicted for future $\mathrm{MHz}$ experiments at EuXFEL using aqueous jets. A corresponding graph displaying the dependencies of the leading shock pressure on the propagation distance is shown in Appendix D.

The lack of shock damage in the $1.1-\mathrm{MHz}$ experiments reported from EuXFEL using lysozyme crystals is consistent with our results because the calculated shock pressures for these experiments are below the damage thresholds (see Table I). For future experiments at EuXFEL (4.5 MHz and $2.25 \mathrm{MHz}$ ) we used (i) the parameters of the experiment by Wiedorn et al. [28], in which the jet will clear the gap with a margin for error at $4.5 \mathrm{MHz}$, and (ii) an experiment which would maximize the shock pressure in a SFX experiment, conducted with pulses of higher total energy from which a larger fraction is absorbed in the jet due to lower photon energies, and with slightly larger jet diameters at the limit of clearing the gap, estimated using the rule of a required jet translation of five jet diameters between consecutive pulses [24]. The first type of experiment is predicted to subject the samples to shock pressures below the lysozyme damage threshold we observed (degradation of resolution at $S / N=5$ ), at both full (4.5 MHz) and half $(2.25 \mathrm{MHz})$ pulse rates. The second type of experiment will exceed the estimated damage threshold at 4.5 MHz, but not at $2.25 \mathrm{MHz}$.

The pressures in Table I were calculated using the average jet diameter, but jets that carry microcrystals can have large fluctuations in the jet diameter. Therefore, we filtered out shots with large fluctuations in the jet diameter (see Appendix C).

A source of uncertainty for the shock pressure predictions in Table I is the size of the X-ray beam, which in our model is assumed to be much smaller than the jet diameter but was larger than that in some experiments. The first results reported from EuXFEL [27,28] used beams larger than the jets, while later experiments [29] used beams smaller than the jet diameters but comparable to them. We expect that XFEL beams smaller but comparable to the jet size will generate weaker shock waves than in the model, therefore, the values in Table I may overestimate the shock pressures.

Among the four different scenarios we investigated for future SFX experiments, only one predicts an observable loss of diffraction quality for lysozyme crystals, at a predicted shock pressure of $55 \mathrm{MPa}$. Based on data in Fig. 3, this pressure corresponds to a resolution drop of $\approx 0.15 \AA$ relative to the reference no-pump data, when analyzing peaks with a signalto-noise ratio above 5 . This degree of shock-induced damage may be acceptable for some projects. Nevertheless, being able to detect shock-induced damage for a particular protein crystal remains important because (i) the crystal may have a lower damage threshold and degrade more when shocked, (ii) the protein structure may change, and (iii) the degree of loss in the diffraction quality that we observed may be unacceptable for some projects. Since the shock damage thresholds are likely to depend on the sample type and since the shock pressure will 
depend on the x-ray absorption of the jet, it remains important to verify experimentally that shock damage does not occur when SFX experiments are conducted at $\mathrm{MHz}$ pulse rates.

Initial SFX experiments conducted at EuXFEL used a 12-hour shift or more acquiring the data needed to search for shock damage because undamaged data were acquired using the first pulse in the trains, which have a $10-\mathrm{Hz}$ rate. Fortunately, if samples must be checked for shock-induced damage, the data in Table I suggest a much more rapid method to test for shock damage: comparing data sets recorded at full rate $(4.5 \mathrm{MHz})$ with half-rate $(2.25 \mathrm{MHz})$, because halving the repetition rate reduces significantly the shock pressure and the extent of the damage. More generally, if diffraction data collected at pulse rates differing by a factor of 2 have the same quality, a decrease in diffraction quality due to shocks is unlikely to be observable. Such data sets can be collected rapidly. Five-minute runs limited in the data rate by the AGIPD detectors at EuXFEL (3250 images/s) [47], at a 1\% crystal hit rate, will provide on the order of 10000 diffraction images, a number that is sufficient for rapid comparisons using the number of all diffraction peaks per image, or for more sensitive comparisons using the maximum diffraction resolution of indexed peaks. Efficient implementation of this procedure requires the existence of a dedicated analysis pipeline that can process rapidly, and then compare, different measures of shock-induced damage.

Compared to EuXFEL, the risk of shock damage during SFX will be much lower at LCLS-II and at SHINE because they have a smaller maximum pulse rate $(1 \mathrm{MHz})$ and lower pulse energies $(<1 \mathrm{~mJ})$ during sustained operation at $1 \mathrm{MHz}[18,19]$. Neverthless, the shocks transmitted in liquid jets might be detrimental in other applications. For example, to maximize the data rate, an x-ray spectroscopy experiment can be performed at $1 \mathrm{MHz}$ with pulses attenuated until they no longer vaporize the liquid, using wide and slow liquid jets ( $\sim 10-100 \mu \mathrm{m}, \sim 10 \mathrm{~m} / \mathrm{s})$. Using the pulse energy threshold for vaporization in water jets [23] and Eq. (1), shocks with estimated peak pressures on the order of $10 \mathrm{MPa}$ will be produced. Due to the smaller shock attenuation in wider jets, these shocks can travel over hundreds of microns [30], and the samples will be subjected to many $~ 10-\mathrm{MPa}$ shocks from previous pulses.

\section{B. Using shock trains in liquid jets to study shock damage in soft matter}

An important application of XFELs is the study of nanosecond structural transformations in materials dynamically compressed to pressures of tens of GPa, using shock waves launched by high-energy lasers $[9,10]$. These explorations of materials transforming at high pressures will soon be extended to more materials and wider timescales by dynamic diamond anvil cell [48] setups at EuXFEL.

The shock waves launched in liquid jets could also be used to study with XFELs the dynamics of materials subjected to transient pressures. Compared to typical dynamic compression studies at XFELs, the shocks launched in liquid jets reach much smaller pressures on the order of $1 \mathrm{GPa}$, but these pressures are sufficient to transform and damage soft matter. Also, the shock trains can produce both positive and negative pressures [25]. Using shocks in liquid jets to apply pressure has the advantage that the experimental setup is relatively simple and the experiments can be conducted at the full pulse rate on a very large number of samples because they are not limited by the number of available fixed-target samples, or by the low repetition rate of the high-energy lasers used for dynamic compression studies.

To be useful for dynamic pressure studies, experiments using shocks in jets should probe the sample at arbitrary times during the pressure pulse, including while the high pressure is applied. This was not possible in our experiments because the two-bunch mode could not be simultaneously set up for sufficiently short delays, different photon energies, and vertical separation. Fortunately, other solutions became possible since our experiments. A simple design is to use a pulsed optical laser to drive the shock trains [30], but further studies are needed to ensure that the optically driven shock trains match the reproducibility and symmetry of XFEL-induced shocks, and it may prove difficult to launch shocks in jets with diameters below $\approx 10 \mu \mathrm{m}$. A better design is to use simultaneously the first-generation LCLS and the second-generation LCLS-II [18], with one XFEL driving the shocks and the second one probing the sample; since these two XFELs are independent, parameters such as the time delay can be adjusted without impacting the XFEL performance.

\section{CONCLUSION}

The observation of shock trains in liquid jets led to concerns about shock-induced sample damage in SFX experiments conducted at second-generation XFELs [23]. Using closely spaced pairs of x-ray pulses at a first-generation XFEL, we observed shock damage in SFX experiments with lysozyme microcrystals. Several measures of diffraction quality were degraded by the shocks: the hit rate, the number of diffraction peaks, the diffraction resolution, the indexing rate, as well as the statistics of the integrated diffraction intensities. We developed formulas for estimating the shock pressures launched by the previous pulse, and we estimated that the shock pressure thresholds for the onset of diffraction degradation in lysozyme are in the tens of megapascals. These pressure thresholds were not exceeded in the first SFX experiments at 1.1-MHz pulse rates, but may be exceeded if EuXFEL is operated at its maximum pulse rate of $4.5 \mathrm{MHz}$.

Our work investigated the behavior of lysozyme crystals, but since the shock tolerance of other samples may be different, it remains prudent to test for the existence of damage in $\mathrm{MHz}$ rate SFX experiments. The dependence of the observable measures of crystal damage on pulse energy, and on the corresponding shock pressure, can be used to design protocols for rapid tests for shock damage during the beam time. Aside from helping design better SFX experiments at $\mathrm{MHz}$ rates, the observation of shock damage in protein crystals enables dedicated XFEL experiments to study the dynamics of shock damage in soft matter.

\section{ACKNOWLEDGMENTS}

The experiments were performed at the Linac Coherent Light Source (LCLS), SLAC National Accelerator 
Laboratory. Use of the LCLS is supported by the U. S. Department of Energy, Office of Science, Office of Basic Energy Sciences under Contract No. DE-AC02-76SF00515. C.A.S. was supported by the U. S. Department of Energy, Office of Science, Chemical Sciences, Geosciences, and Biosciences Division, and by startup funds from Rutgers University Newark. Part of the sample injector used at LCLS for this research was funded by the National Institutes of Health, P41GM103393, formerly P41RR001209. We thank Y. Aulin and P. Piotrowiak for assistance in testing the diode system for the 122.5-ns experiment.

\section{APPENDIX A: SETUP AND CHARACTERIZATION OF THE XFEL TWO-BUNCH MODE}

The "two-bunch" mode used in the experiments is based on producing two XFEL pulses from two electron clouds ("bunches") that are generated and accelerated separately [31]. The LCLS linear accelerator uses a pulsed radio-frequency (rf) field at $2.856 \mathrm{GHz}$ (350.14-ps period) filling the accelerating structure for $825 \mathrm{~ns}$. The multiple wave peaks in the accelerating field (or "buckets") can each accelerate electron bunches. All these bunches can, in principle, achieve $\mathrm{x}$-ray lasing if they are temporally spaced by multiples of the accelerating field period of $350 \mathrm{ps}$; in our experiments the pulses were separated by $8.4 \mathrm{~ns}$ ( 24 buckets spacing) and 122.5 ns (350 buckets). Both pulses had similar properties to the pulses from a standard experiment with hard $\mathrm{x}$ rays at LCLS $(\approx 50$-fs duration, on the order of $1 \mathrm{~mJ}$ total pulse energy).

The two XFEL pulses had different photon energies, with a separation of $\approx 80 \mathrm{eV}$ centered on the iron $\mathrm{x}$-ray absorption $\mathrm{K}$ edge at $7.11 \mathrm{keV}$. The first pulse had the photon energy above the edge, and the second below the edge. The pulses were generated at different vertical positions by making the two electron bunches travel at different heights in the undulator at the photon source point. The vertically separated XFEL pulses at the source were imaged by the x-ray focusing mirror onto two different positions along the jet. At the jet, the separation between pulses was $\approx 5 \mu \mathrm{m}$, with the first pulse arriving vertically below the second pulse.

Generation of two separate electron bunches. For both experiments, two distinct electron clouds were produced at the gun photocathode by separate laser pulses produced by two injector lasers. Using two lasers simplified the setting of the time delay between pulses.

Generating pulses with different photon energies. The different $\mathrm{x}$-ray photon energies for the two pulses were obtained by using electron bunches with different kinetic energies. This was achieved by setting the timing of the radio-frequency (rf) accelerating pulse such that the accelerating fields were different for the two bunches. For example, in the 8.4-ns experiment the rf pulse was advanced by 50 ns relative to its nominal timing, such that the envelope of the rf field was decaying and the first bunch experienced higher accelerating fields leading to a larger kinetic energy.

The width of spectral distributions of pump and probe pulses was comparable to the separation between their mean photon energies, therefore, a small fraction of the probe photons had photon energies above the iron edge, and a small fraction of the pump photons had photon energies below the iron edge. The latter category will lead to diffraction that is not blocked by the iron filter and may lead to an underestimate of the damage because diffraction patterns from the pump may be included in the data, but this effect was small. A survey of the spectrometer data in the 8.4-ns experiment indicated that at most $10 \%$ of the pump photons, but more usually on the order of $1 \%$, had energies below the edge. For low pump energies the contribution to the diffraction patterns is even smaller since the probe pulse had more photons. In the 122.5-ns experiment the Fe foil masked diode was used to remove from analysis shots in which the energy of pump photons below the $\mathrm{Fe}$ edge exceeded $\approx 2 \%$ of the average probe energy (see Appendix $\mathrm{C}$ for diffraction data filtering).

Vertical separation of the pulses. The separation of the XFEL pump and probe beams was achieved by vertically separating the electron bunches in the undulator. Two methods were used in combination to separate the bunches: (i) Applying a time-varying transverse electric field such that the two bunches were deflected by different amounts. This was done inside one of the transverse cavities in the accelerator (TCAV3). (ii) By inducing vertical dispersion with respect to the electron energy in the undulator. Ideally, the dispersion affects only the vertical position at the photon source point, so the different energy bunches will create photons at different vertical positions. In the 8.4-ns experiment the primary separation was done by the transverse field, and dispersion was used for correction. In the 122.5-ns experiment it was all done by vertical dispersion.

Manipulation of the vertical position of the electron bunches led to separation of the XFEL beams both in position and in angle at the generation location (i.e., the XFEL "source"). The vertical separation at the sample depends only on the vertical separation at the source because the source is imaged onto the sample by the focusing mirrors. The angular separation must be kept small enough such that the beams are not clipped or blocked inside the beam line, however, a small amount of angular separation was useful to control the relative intensities of the two pulses (see below for the tuning the pulse energies). In the 8.4-ns experiment the angular separation was approximately $7 \mu \mathrm{rad}$. In the 122.5 -ns experiment the separation was not measured, but based on imaging the pump and probe beams at the entrance of the focusing mirrors we estimate that the angular separation was about the same as the one in the 8.4-ns experiment.

The transverse field used to separate the bunches vertically increases the jitter of the vertical position of the beam from $\approx 5 \%$ to $\approx 25 \%$ of the beam's root-mean-square (rms) width. However, since both bunches are deflected by the same rf pulse the relative vertical separation of the beams remains the same. As a rule of thumb, the variation in the separations induced by the transverse cavity in time, energy, or transverse position is given by the time separation between pulses divided by rf fill time which is typically $825 \mathrm{~ns}$. Thus, for the 122.5-ns experiment we estimate that the vertical separation jitter is only about $15 \%$ of the overall jitter, or $4 \%$ of the beams' rms width; correspondingly, in the 8.4-ns experiment the vertical separation jitter was less than $1 \%$ of the rms beam width. 
Tuning of the total pulse energies. The XFEL pulse energies could be adjusted over a limited range by changing the intensity of the injector lasers on the gun photocathode, which varied the number of electrons in the bunches. A second adjustment of the pulse energies was performed by first increasing the angular separation of the beams until they could not both fit inside the aperture of the x-ray focusing mirrors. Then, by changing the vertical pointing of the whole undulator, the beams were clipped by different amounts by the mirror aperture, leading to changes in the relative number of pump and probe photons arriving at the sample.

We note that in the two-bunch mode with vertically separated pulses the energies of the two pulses may be anticorrelated. Due to the requirement to achieve a large vertical separation, in the undulator both electron bunches passed on opposite sides of the trajectory that produces the most intense lasing. The lasing intensity has an approximately Gaussian distribution with the vertical position of the electron trajectory, and the bunches were on opposite sides of the peak of the distribution. When the common jitter moved one pulse towards the peak, the other fell further off, anticorrelating the two pulse energies.

Focus size and shape. Due to the unusual operation mode with a transverse offset, source size and divergence may be larger than in standard single-pulse experiments, leading to a larger beam size at the sample than the nominal $1.5-\mu \mathrm{m}$ full width at half-maximum. In addition, to enhance the vertical separation at the sample the longitudinal position of the source was shifted to generate more separated (but less tightly focused) beams. Last, the 8.4-ns experiment used LCLS's original hard x-ray offset mirrors, which had a slight deformation which generated a two- or three-lobed intensity distribution in the horizontal in the far field. This deformation led to a non-perfect focus which was further degraded by the shift in the source position, and we observed jet explosion patterns consistent with two intensity lobes in the vertical direction.

Changes in the beam properties during the probe-only reference runs. As a reference for the x-ray pump x-ray probe data, data were also collected in single-bunch runs ("probe-only" runs). For this purpose the pump pulse (the first of the two pulses) was suppressed by blocking the first injector laser pulse. To prevent automatic adjustment of the single-pulse photon energy to the mean photon energy of the two pulses and to prevent shifting of the spatial location of the single pulse, beam feedbacks were turned off during these runs. Only short runs were taken $(<5 \mathrm{~min})$ such that any drifts of the photon energy and especially of the peak current were small. In the probe-only runs the electron bunch of the probe beam no longer experienced wakefields from the first bunch and had a slightly different orbit, leading to a different lasing intensity in the probe-only runs.

Due to the missing wakefields during the probe-only runs, the photon energy of the probe pulse may slightly increase, thus drifting towards the Fe absorption edge. The wakefields, aside from a main transverse effect, also have a small longitudinal effect on the second bunch. When the first bunch is turned off, a small beam loading effect $(40 \mathrm{~V} / \mathrm{pC} \mathrm{m})$ increases slightly the photon energy. For a bunch charge of $150 \mathrm{pC}$ and an accelerator length of $1000 \mathrm{~m}$, the beam loading effect corresponds to $\approx 6 \mathrm{MeV}$, i.e., $0.05 \%$ of the $12.5-\mathrm{GeV}$ electron energy, and consequently to $0.10 \%$ of the $7.07-\mathrm{keV}$ photon energy. The absolute shift in photon energy is therefore low, $\approx 7 \mathrm{eV}$ towards higher energies.

Pulse energy measurement. The energies of the two x-ray pulses were determined by a measurement system of fast response photodiodes and high-rate digitizers, and had sufficient temporal resolution to temporally resolve the two x-ray pulses. In both experiments, the diode used for pulse energy measurements generated two voltage pulses whose amplitudes, after background subtraction and removal of traces with large electronic noise, were proportional to the energy of the pump and probe pulses. The relative pulse energy measurements were calibrated to absolute energy values at the XFEL source by recording the diode signal in single-pulse experimental runs. For the calibration runs the beam was realigned to achieve the best possible beam-line transmission, such that the transmission was similar to the one in a standard, single-pulse experiment.

For the 122.5-ns experiments the relative x-ray pulse energy was determined by measuring the diffuse scattering $\approx 30 \mathrm{~mm}$ away from a Kapton sheet (four stacked sheets, $510 \mu \mathrm{m}$ total thickness) at approximately $20^{\circ}$ from the beam. The scattered photons were detected by two photodiodes with 30-ps response time (G4176-03, Hamamatsu Photonics K. $\mathrm{K}$.). The diodes were reverse biased at $9 \mathrm{~V}$ for a fast response, using bias tees (ZBFT-4R2G+, Mini-Circuits). The diode signals were first amplified using broadband amplifiers (ZKL-1R5+, Mini-Circuits) and then recorded by two separate multichannel digitizers (U1065A-004, Keysight) at a rate of $8 \mathrm{GHz}$ for each signal. One of the photodiodes, directly exposed to $\mathrm{x}$ rays, recorded both pulses with equal sensitivity and provided the pulse energy measurements. The other photodiode was placed behind a $25-\mu \mathrm{m}$-thick Fe foil (FE000240, Goodfellow Corporation) which absorbed the first pulse if its photon energy was above the $\mathrm{Fe}$ absorption edge. This diode was used to confirm that the first pulse did not have a detectable $\mathrm{x}$-ray spectrum below the absorption edge, which would lead to recording diffraction patterns from the pump pulse. The diode masked by the foil could detect leaked pump photons if their total energy exceeded $\approx 2 \%$ of the average energy of the probe pulse.

The 8.4-ns experiments used a different diode setup, with one diode (the "beam-stop diode") collecting $\mathrm{x}$ rays backscattered at $150^{\circ}$ from the beam by a silica lens that was also used as a beam stop. Silica was used because it darkens after XFEL exposure; the dark spot on the lens was used to align the diode below the beam and $\approx 25 \mathrm{~mm}$ from the dark spot. This diode was used to measure the pulse energies. Another diode (the "jet diode"), placed $\approx 40 \mathrm{~mm}$ away from the jet, recorded the $\mathrm{x}$ rays backscattered at $150^{\circ}$. The photodiodes used in the 8.4-ns experiment had a 700-ps response time (AXUVHS11, Opto Diode Corp) and were reverse biased at $\approx 40 \mathrm{~V}$ using bias tees (PSPL5575A, Tektronix, Inc.). The diode signals were amplified (backstop diode: ZFL-1000LN+, Mini-Circuits; jet diode: C5594-22, Hamamatsu Photonics K. $\mathrm{K}$.), and recorded by a single multichannel digitizer (U1065A004, Keysight) at a rate of $4 \mathrm{GHz}$ for each signal. 


\section{APPENDIX B: CRYSTAL PREPARATION AND SAMPLE DELIVERY}

Preparation of lysozyme microcrystals. Microcrystals $(1 \times$ $1 \times 3 \mu \mathrm{m}^{3}$ ) of hen egg-white lysozyme (Sigma-Aldrich Chemie GmbH, Schnelldorf, Germany) were grown by rapidly mixing cold solutions of protein $(32 \mathrm{mg} / \mathrm{ml}$ in $0.2 \mathrm{M}$ $\mathrm{Na}$ acetate $\mathrm{pH} 3.0)$ and precipitant $(20 \% \mathrm{NaCl}, 6 \%$ PEG 6000 , $1 \mathrm{M} \mathrm{Na}$ acetate $\mathrm{pH}$ 3.0) in a $1: 3$ ratio in a $4{ }^{\circ} \mathrm{C}$ cold room as described previously [49]. After overnight crystallization, the microcrystals were washed with storage solution $(10 \% \mathrm{NaCl}$, $0.1 \mathrm{M} \mathrm{Na}$ acetate $\mathrm{pH} 4.0$ ).

Crystal delivery into the XFEL beam. Lysozyme microcrystals suspended in their aqueous storage solution were injected into the $\mathrm{x}$-ray interaction region via a liquid microjet produced by gas dynamic virtual nozzles (GDVN) [22] with 75- and $100-\mu \mathrm{m}$ inner diameters using helium as the focusing gas. The sample flow rate was $30-50 \mu \mathrm{l} / \mathrm{min}$. The gas pressure was 300-600 psi at the inlet of the 2-m-long, 100- $\mu \mathrm{m}$ inner diameter GDVN gas supply line. The jets had a diameter of $\approx 5 \mu \mathrm{m}$ and a velocity of $\approx 50 \mathrm{~m} / \mathrm{s}$. All samples were filtered prior to injection through a $20-\mu \mathrm{m}$ filter, and the suspension was adjusted to contain 10\%-20\% (v/v) settled crystalline material. To prevent the settling of crystals, the sample was continuously rotated end over end in a reservoir temperature controlled at $20^{\circ} \mathrm{C}[50]$.

Jet imaging. The jet was imaged optically using a microscope with femtosecond laser illumination. The liquid jet was imaged orthogonal to both $\mathrm{x}$ rays and jet flow direction using a $50 \times$ infinity-corrected objective (Plan Apo SL, Mitutoyo Corporation) in combination with a $200-\mathrm{mm}$ tube lens. We split the image in two copies projected onto (i) a camera (Opal 1000 , Adimec) that recorded images for every pulse to verify the overlap of the jet and the pump pulse, and (ii) a high-speed camera (Vison Research, Miro R341) used for measurements of the jet velocity and of the vertical separation between the pump and probe pulses. The optical resolution of the imaging system was determined to be better than $780 \mathrm{~nm}$, using USAF 1951 resolution targets (Edmund Optics, Ready Optics). To prevent motion blurring the femtosecond optical pump laser at CXI [34] was employed for jet illumination [23]. The femtosecond laser pulse and the camera were triggered by electronic signals synchronized with the x-ray pulses, such that the images were recorded at a set delay relative to the arrival of the X-ray pulses. In the 8.4-ns experiment the jet was imaged at the time when the probe pulse arrived to capture the jet configuration when it was probed. In the 122.5-ns experiment the jet was imaged a few nanoseconds after the probe pulse, to observe the formation of two distinct gaps in the jet.

Measurement of the jet velocity and the beam separation. Microjet velocities can be measured by tracking a jet feature in time [51]. In our case, the tracked feature was the center of the gap produced by the XFEL interaction with the jet, which due to the symmetry of the explosion translates at the same speed as the jet [23]. We imaged the pump-induced gap in the jet at two different imaging delays $\Delta t$ relative to the arrival of the pump, and we measured the distance $d$ the gap center has moved downstream. The jet velocity $v_{\text {jet }}$ was calculated as $v_{\text {jet }}=d / \Delta t$. The beam separation was then determined by subtracting the jet displacement between pulses (i.e., $v_{\text {jet }} \Delta t_{\text {pair }}$; see Fig. 1) from the separation of the gaps made by the pump and probe pulses.

\section{APPENDIX C: DIFFRACTION DATA PROCESSING}

Crystallographic data processing and structure refinement. CASS [42,43] was used for online data analysis, hit identification, and data preprocessing. The positions and orientations of individual sensor modules of the CSPAD were refined as previously described [6]. Diffraction images were used for analysis if they passed several filtering criteria (see paragraph below for filtering). Diffraction peaks were identified by CRYSTFEL [52] from calibrated detector images that had passed filtering using the gradient search after Zaefferer, with the following peak detection parameters: - threshold $=100-$ min-snr $=4-$ no-use-saturated - min-gradient $=1000$ for the $8.4 \mathrm{~ns}$ data, and - threshold $=30$ - min-snr $=5-$ min-gradient $=10000$ for the $122.5 \mathrm{~ns}$ data. Indexing and integration of the filtered diffraction images were performed with CRYSTFEL [52] version 0.8.0 using the xgandalf, dirax, and mosflm indexers with the no-cellcombinations option. The unit-cell constants did not differ significantly between the pump-only and the pump-probe data (see Supplemental Material [44]). The resolution of a diffraction pattern was determined from the indexed diffraction peak with the highest diffraction angle among the peaks with a signal-to-noise ratio above a preset value; to check that the resolution was not influenced by detector shadowing, we extracted two resolution data sets at signal-to-noise ratios of 4 and 5 and verified that they had a similar trend. The probeonly and pump-probe SFX data of lysozyme were phased by molecular replacement using REFMAC version 5.8.0222 [53] and PDB entry 4ET8 as a search model after removal of water molecules. The final structures were obtained using iterative cycles of rebuilding in COOT [54] and refinement in REFMAC [53]. Data statistics are available as Supplemental Material [44].

Structural comparison. To maximize the accuracy of crystal-structure determination, and the sensitivity for detecting possible radiation damage, the crystal structures were determined using all the images that passed the filtering steps, independent of probe-pulse energies. Data statistics for this analysis are available as Supplemental Material [44].

The pump-only and pump-probe structures were compared using approaches described previously for analyzing structural changes induced by static pressure [39]. We detected no significant conformational changes.

Test for radiation damage. The integrity of disulfide bonds is a sensitive marker for radiation damage. To test whether the observed changes in diffraction data quality might have been caused by radiation damage by stray $\mathrm{x}$-ray light from the pump pulse, we calculated difference electron density maps. To this end, after indexing, stream files for difference map calculations were truncated to contain the same number of images for both the potentially damaged and the reference data sets. Both data sets were then integrated according to the Monte Carlo scheme [55] and scaled to each other using SCALEIT [56], including Wilson scaling. Difference density maps were calculated with FFT [56] and 
displayed in COOT [54,57]. No significant features were observed.

Diffraction data filtering. Due to hydrodynamic instabilities, the jet position and shape fluctuated, and in the 122.5-ns experiment the jet was not always hit by both pulses. If a jet breakup occurs between the regions hit by the pump and the probe pulses, the shockwave launched by the pump cannot travel to the probed region. Therefore, optical images recorded a few nanoseconds after the second pulse were used to select for analysis only shots in which the pump pulse hit the jet and the jet segment between the two interaction points was continuous; since the crystal hits require that the probe interacts with the jet, the data were effectively filtered to contain only shots in which both pulses hit the jet. A Python 3.7 script was written to determine indicators of jet shape and the number of gaps in the region of the jet where the pump and probe pulses arrived. For analysis, images were selected in which the projected jet size was within one standard deviation of the median projected size within a given run (thereby excluding overly thin, thick, or skew jets in which shock wave magnitude and propagation differ), in which XFEL-induced gaps were visible, and in which the jet segment upstream of the pump region is continuous, allowing shock wave propagation to the point of probe. In the 8.4-ns experiment this analysis was not necessary because the fraction of shots hit by only one of the pulses was very small.

Shots with probe-pulse energies smaller than approximately half, and larger than approximately twice, the mean probe energy, comprising approximately one quarter of the data, were removed from analysis. Shots with low probe-pulse energies are affected by large measurement errors, while shots with high energies sometimes had additional off-nominal properties such as their photon energy.

We also removed from analysis shots in which the total energy of pump photons leaking below the Fe edge, measured by the Fe foil masked diode, exceeded $\approx 2 \%$ of the average probe-pulse energy.

Binning of data by the pump-pulse energy. The properties of the diffraction patterns had wide distributions, most likely due to the varying degrees of overlap between the crystals and the XFEL beam. To enhance the measurement sensitivity, the data were binned by the pump energy, and the reported data are the mean value across each bin. Also, Figs. 2 and 3 show the standard deviation of the mean values, which were calculated as the standard deviation of individual shots divided by the square root of the number of shots in the bin. For the 122.5-ns experiment the bins had uneven widths to improve the resolution of the pressure dependence. For the 8.4-ns experiment we used a large and uniform bin size because the deconvolution of the photodiode noise (see below) required few bins with constant sizes.

Equalization of the probe-pulse energies. Since the intensity of the diffraction peaks increases with the total energy of the probe pulse, a meaningful comparison between pumpprobe and probe-only data requires selecting for analysis shots that have the same probe-pulse energy. The amount of data in our experiments was not sufficient for selecting shots with a narrow and matching range of probe energies. Instead, we equalized the distribution of the probe energies to a flat-top distribution over a broad range of pulse energies; this was

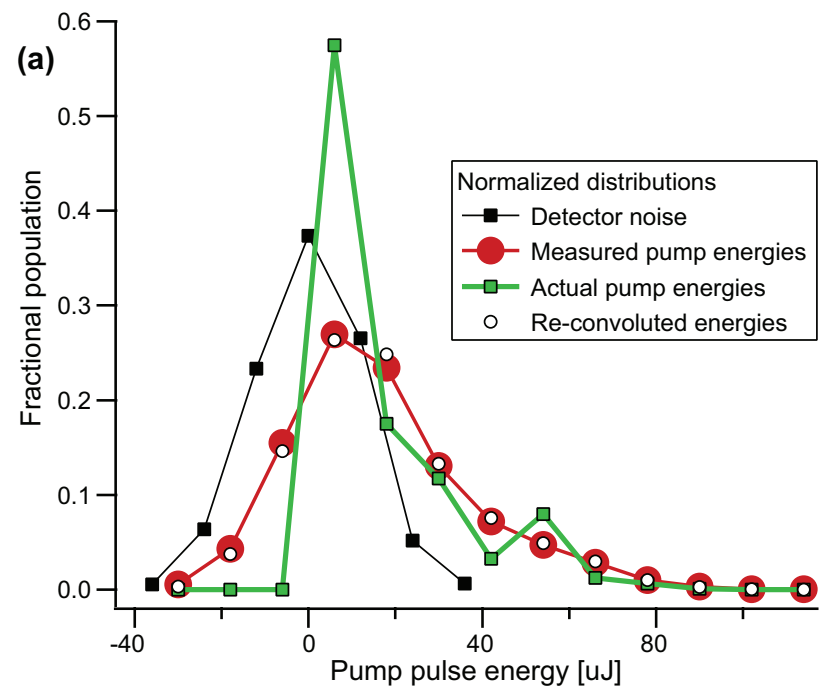

(b)

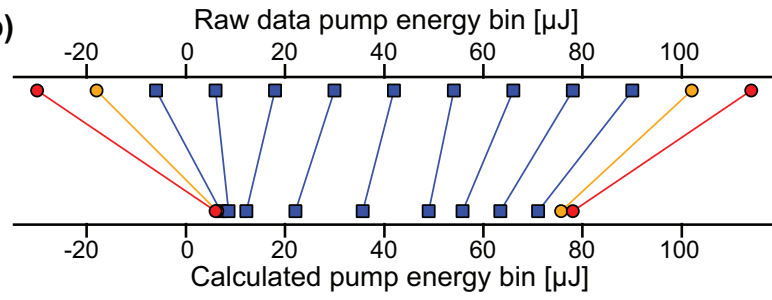

FIG. 4. Deconvolution analysis of the pump energy measurements in the 8.4-ns experiment. (a) The diode detector noise distribution was deconvolved from the distribution of measurements to derive the actual distribution of pump energies. (b) The actual values of the energy bins shown in relation to measured bin centers. The first and last two bin positions (red and orange symbols) have a large dependence on the chosen bin size and have not been included in the final data analysis.

done for all the bins in the pump energy and for the probeonly data. We first removed shots that had very low or very high probe energies, as described in the data-filtering section above. Then, the remaining data were divided into four equally wide bins in the probe energy, and the numbers of shots were made equal in all bins by randomly removing shots from the bins containing more shots than the others. After the equalization, the average probe energies in all data sets used in analysis had values within $1 \%$ of each other.

Pump-pulse energy deconvolution analysis for the 8.4-ns experiment. In the 8.4-ns experiment, the noise of the photodiode was comparable to the measured signal. Figure 4(a) shows the distribution of pump-pulse readings from probe-only measurements (in which the pump-pulse energy was zero). This is the detector noise distribution, and its width is almost as large as the width of the distribution of pulse energy measurements from the pump-probe data. While the photodiode readings for the pump pulse can be binned using the raw data, pressures cannot be calculated correctly using the negative raw pump energy values. To calculate the shock pressure from bins with negative raw energies, we calculated the actual energy of the bins using the actual distribution of pump energies, which was determined by deconvolving the detector noise from the 
distribution of raw pump energy readings. The deconvolution analysis was not needed for the 122.5-ns experiment because the photodiode readings had a much better signalto-noise ratio, and the raw bin energies were sufficiently accurate.

The calculated energies of the bins are shown in Fig. 4(b) along with the raw values of the bins. The calculated energies no longer have unphysical negative values; for example, we found that the data bin centered at $-6 \mu \mathrm{J}$ in the raw data had an actual average pump energy of $8 \mu \mathrm{J}$. However, the smallest and largest calculated bin energies depend on the choice of the bin width and were not used for further analysis.

If the detector noise distribution is precisely known, the true signal can be calculated using an exact deconvolution algorithm, but exact algorithms could not be applied due to the finite precision of the measurements. Instead, the deconvolution was performed iteratively using the approximate Lucy-Richardson (LR) deconvolution algorithm, in the form implemented in MATLAB R2018a. After 100 iterations the LR algorithm converged to solution with a narrower distribution of energy values. To determine the actual distribution of pump values shown in Fig. 4 we imposed in the LR solution zero values for bins with negative energies, and we verified the actual distribution by testing that its convolution with the detector noise (i.e., the reconvoluted distribution) reproduced the experimental distribution.

The actual bin energies $E_{m}$, where $m$ is the bin index in the measured data, were calculated according to Eq. (C1):

$E_{m}=\frac{\sum_{d \geqslant \max \left(1, m+1-N_{A}\right)}^{d \leqslant \min \left(N_{D}, m\right)} E_{m-d+1}^{A} P_{D}(d) P_{A}(m-d+1)}{\sum_{d \geqslant \max \left(1, m+1-N_{A}\right)}^{d \leqslant \min \left(N_{D}, m\right)} P_{D}(d) P_{A}(m-d+1)}$,

where $d$ is the bin index for the detector noise, $N_{D}$ and $N_{A}$ are the total number of the bins in the detector noise curve and the in the actual (i.e., deconvoluted) pulse energy distribution, $E_{k}^{A}$ is the energy of the $k$ th bin in the actual pulse energy distribution, and $P_{D}$ and $P_{A}$ are the discrete distribution functions for the detector noise and the actual pulse energy. The denominator in Eq. (C1) is the (not-normalized) reconvoluted distribution.

\section{APPENDIX D: DECAY OF THE SHOCK PRESSURE ACCORDING TO THE MODEL, AND ITS COMPARISON WITH EXPERIMENTAL DATA}

Figure 5(a) displays measurements of the pressure of the leading shocks generated by $9.53-\mathrm{keV} \mathrm{x}$ rays in water jets [25], along the predictions of Eq. (1). The model overestimates slightly the measured pressures, but the measurements and predictions are within a factor of 2 of each other for most data. The logarithmic pressure scale may also overemphasize the differences if the experimental data have high noise.

Figure 5(b) shows the dependence of the model on $\Delta y_{\text {shock }}$ (the distance along the jet between regions hit by consecutive pulses), for the experiments and scenarios listed in Table I. The graph illustrates several properties of the model. The short-distance part of the decay of the pressure with $\Delta y_{\text {shock }}$ is
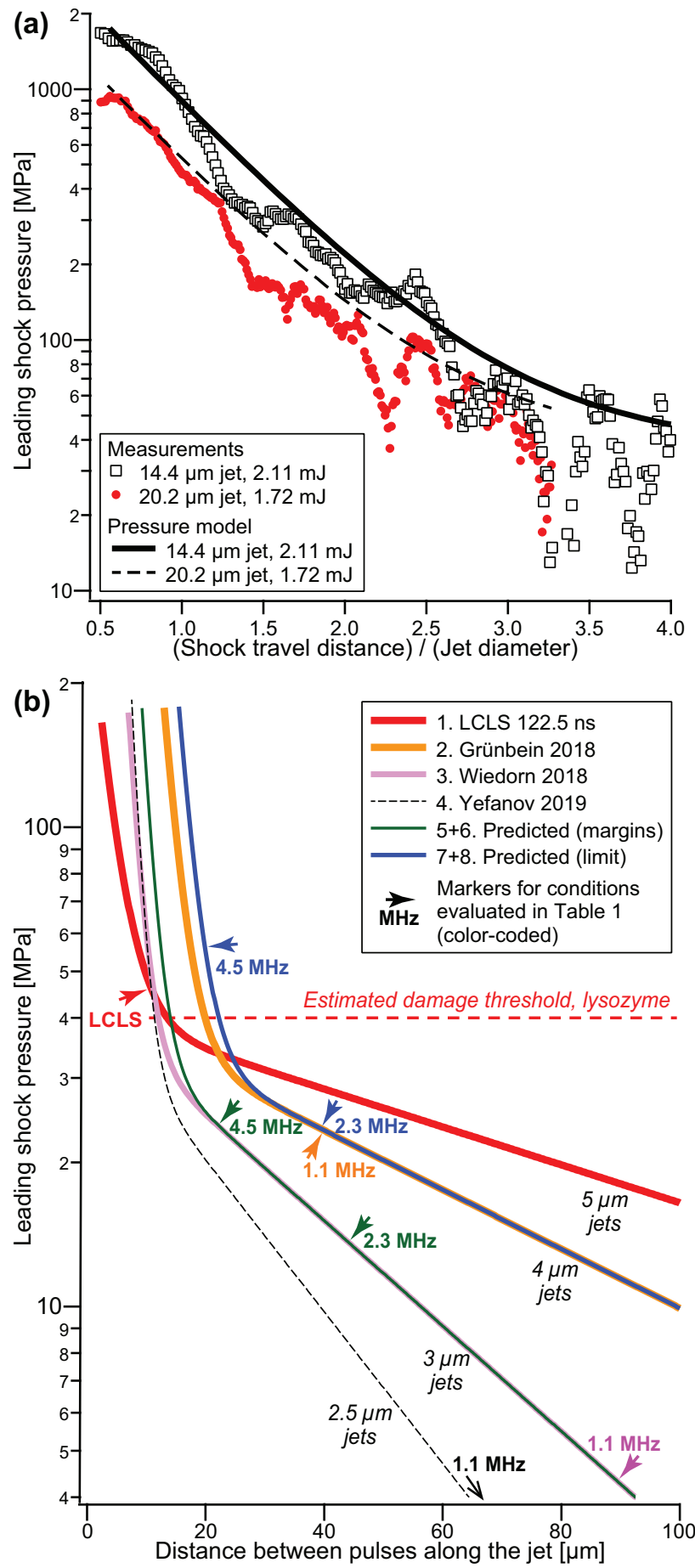

FIG. 5. (a) Comparison between shock pressure measurements from Ref. [25] and the predictions of Eq. (1). (b) The decay of the shock pressure predicted by Eq. (1) for the conditions listed in Table I. The arrow markers indicate the distances between regions hit by consecutive pulses, for the corresponding jet velocities and pulse rates in Table I.

the most rapid, and the pressures have a strong dependence on the pulse energy (i.e., the curves are distinct for jets with same diameter but different energies). The long-distance part of the decay is slower, and the pressures are almost independent of the initial pulse energy (i.e., the curves overlap for jets with the same diameter). The transition between these regimes 
occurs at pressures close to the long-lived shock pressure $P_{\text {long }}=40 \mathrm{MPa}$, and the weak long-distance dependence of pressure on the pulse energy is due to the finite value of $P_{\text {long }}$. The long-distance dependence on $\Delta y_{\text {shock }}$ also illustrates the strong dependence of the shock decay on the jet diameter. Al- though the long-distance dependence of pressure on $\Delta y_{\text {shock }}$ is less steep than at short distances, it is exponential; increasing $\Delta y_{\text {shock }}$ by a factor of 2 , which can be achieved by doubling the jet velocity or by halving the pulse rate, leads to a decay of the pressure of at least $30 \%$.
[1] P. Emma, R. Akre, J. Arthur, R. Bionta, C. Bostedt, J. Bozek, A. Brachmann, P. Bucksbaum, R. Coffee, F. J. Decker, Y. Ding, D. Dowell, S. Edstrom, A. Fisher, J. Frisch, S. Gilevich, J. Hastings, G. Hays, P. Hering, Z. Huang et al., First lasing and operation of an ångström-wavelength free-electron laser, Nat. Photonics 4, 641 (2010).

[2] T. Ishikawa, H. Aoyagi, T, Asaka, Y. Asano, N. Azumi, T. Bizen, H. Ego, K. Fukami, T. Fukui, Y. Furukawa, S. Goto, H. Hanaki, T. Hara, T. Hasegawa, T. Hatsui, A. Higashiya, T. Hirono, N. Hosoda, M. Ishii, T. Inagaki et al., A compact x-ray free-electron laser emitting in the sub-ångström region, Nat. Photonics 6, 540 (2012).

[3] H. N. Chapman, P. Fromme, A. Barty, T. A. White, R. A. Kirian, A. Aquila, M. S. Hunter, J. Schulz, D. P. DePonte, U. Weierstall, R. B. Doak, F. R. N. C. Maia, A. V. Martin, I. Schlichting, L. Lomb, N. Coppola, R. L. Shoeman, S. W. Epp, R. Hartmann, D. Rolles et al., Femtosecond X-ray protein nanocrystallography, Nature (London) 470, 73 (2011).

[4] M. M. Seibert, T. Ekeberg, F. R. N. C. Maia, M. Svenda, J. Andreasson, O. Jonsson, D. Odic, B. Iwan, A. Rocker, D. Westphal, M. Hantke, D. P. DePonte, A. Barty, J. Schulz, L. Gumprecht, N. Coppola, A. Aquila, M. N. Liang, T. A. White, A. Martin et al., Single mimivirus particles intercepted and imaged with an X-ray laser, Nature (London) 470, 78 (2011).

[5] K. H. Kim, J. G. Kim, S. Nozawa, T. Sato, K. Y. Oang, T. Kim, H. Ki, J. Jo, S. Park, C. Song, T. Sato, K. Ogawa, T. Togashi, K. Tono, M. Yabashi, T. Ishikawa, J. Kim, R. Ryoo, J. Kim, $\mathrm{H}$. Ihee, and S. Adachi, Direct observation of bond formation in solution with femtosecond x-ray scattering, Nature (London) 518, 385 (2015).

[6] T. R. M. Barends, L. Foucar, A. Ardevol, K. Nass, A. Aquila, S. Botha, R. B. Doak, K. Falahati, E. Hartmann, M. Hilpert, M. Heinz, M. C. Hoffmann, J. Kofinger, J. E. Koglin, G. Kovacsova, M. Liang, D. Milathianaki, H. T. Lemke, J. Reinstein, C. M. Roome et al., Direct observation of ultrafast collective motions in $\mathrm{CO}$ myoglobin upon ligand dissociation, Science 350, 445 (2015).

[7] J. M. Glownia, A. Natan, J. P. Cryan, R. Hartsock, M. Kozina, M. P. Minitti, S. Nelson, J. Robinson, T. Sato, T. van Driel, G. Welch, C. Weninger, D. Zhu, and P. H. Bucksbaum, SelfReferenced Coherent Diffraction X-Ray Movie of Angstromand Femtosecond-Scale Atomic Motion, Phys. Rev. Lett. 117, 153003 (2016).

[8] D. Milathianaki, S. Boutet, G. J. Williams, A. Higginbotham, D. Ratner, A. E. Gleason, M. Messerschmidt, M. M. Seibert, D. C. Swift, P. Hering, J. Robinson, W. E. White, and J. S. Wark, Femtosecond visualization of lattice dynamics in shockcompressed matter, Science 342, 220 (2013).

[9] M. G. Gorman, R. Briggs, E. E. McBride, A. Higginbotham, B. Arnold, J. H. Eggert, D. E. Fratanduono, E. Galtier, A. E. Lazicki, H. J. Lee, H. P. Liermann, B. Nagler, A. Rothkirch, R. F. Smith, D. C. Swift, G. W. Collins, J. S. Wark, and
M. I. McMahon, Direct Observation of Melting in ShockCompressed Bismuth With Femtosecond X-ray Diffraction, Phys. Rev. Lett. 115, 095701 (2015).

[10] A. E. Gleason, C. A. Bolme, H. J. Lee, B. Nagler, E. Galtier, D. Milathianaki, J. Hawreliak, R. G. Kraus, J. H. Eggert, D. E. Fratanduono, G. W. Collins, R. Sandberg, W. Yang, and W. L. Mao, Ultrafast visualization of crystallization and grain growth in shock-compressed $\mathrm{SiO}_{2}$, Nat. Commun. 6, 8191 (2015).

[11] C. A. Stan, P. R. Willmott, H. A. Stone, J. E. Koglin, M. Liang, A. L. Aquila, J. S. Robinson, K. L. Gumerlock, G. Blaj, R. G. Sierra, S. Boutet, S. A. H. Guillet, R. H. Curtis, S. L. Vetter, H. Loos, J. L. Turner, and F. J. Decker, Negative pressures and spallation in water drops subjected to nanosecond shock waves, J. Phys. Chem. Lett. 7, 2055 (2016).

[12] J. A. Sellberg, C. Huang, T. A. McQueen, N. D. Loh, H. Laksmono, D. Schlesinger, R. G. Sierra, D. Nordlund, C. Y. Hampton, D. Starodub, D. P. DePonte, M. Beye, C. Chen, A. V. Martin, A. Barty, K. T. Wikfeldt, T. M. Weiss, C. Caronna, J. Feldkamp, L. B. Skinner et al., Ultrafast X-ray probing of water structure below the homogeneous ice nucleation temperature, Nature (London) 510, 381 (2014).

[13] A. J. Amaya, H. Pathak, V. P. Modak, H. Laksmono, N. D. Loh, A. S. Jonas, R. G. Sierra, T. A. McQueen, M. J. Hayes, G. J. Williams, M. Messerschmidt, S. Boutet, M. J. Bogan, A. Nilsson, C. A. Stan, and B. E. Wyslouzil, How cubic can ice be? J. Phys. Chem. Lett. 8, 3216 (2017).

[14] R. Neutze, R. Wouts, D. van der Spoel, E. Weckert, and J. Hajdu, Potential for biomolecular imaging with femtosecond X-ray pulses, Nature (London) 406, 752 (2000).

[15] S. Boutet, L. Lomb, G. J. Williams, T. R. M. Barends, A. Aquila, R. B. Doak, U. Weierstall, D. P. DePonte, J. Steinbrener, R. L. Shoeman, M. Messerschmidt, A. Barty, T. A. White, S. Kassemeyer, R. A. Kirian, M. M. Seibert, P. A. Montanez, C. Kenney, R. Herbst, P. Hart et al., High-resolution protein structure determination by serial femtosecond crystallography, Science 337, 362 (2012).

[16] C. Bostedt, S. Boutet, D. M. Fritz, Z. R. Huang, H. J. Lee, H. T. Lemke, A. Robert, W. F. Schlotter, J. J. Turner, and G. J. Williams, Linac Coherent Light Source: The first five years, Rev. Mod. Phys. 88, 015007 (2016).

[17] W. Decking, S. Abeghyan, P. Abramian, A. Abramsky, A. Aguirre, C. Albrecht, P. Alou, M. Altarelli, P. Altmann, K. Amyan, V. Anashin, E. Apostolov, K. Appel, D. Auguste, V. Ayvazyan, S. Baark, F. Babies, N. Baboi, P. Bak, V. Balandin et al., A MHz-repetition-rate hard X-ray free-electron laser driven by a superconducting linear accelerator, Nat. Photonics 14, 391 (2020).

[18] P. Abbamonte, F. Abild-Pedersen, P. Adams, M. Ahmed, F. Albert, R. Alonso-Mori, P. Anfinrud, A. Aquila, M. Armstrong, J. Arthur, J. Bargar, A. Barty, U. Bergmann, N. Berrah, G. Blaj, H. Bluhm, C. Bolme, C. Bostedt, S. Boutet, G. Brown et al., New Science Opportunities Enabled by LCLS-II X-ray Lasers, 
Report SLAC-R-1053 (SLAC National Accelerator Laboratory, Menlo Park, CA, 2015).

[19] N. Huang, H. Deng, B. Liu, and D. J. Wang, Physical design and FEL performance study for FEL-III beamline of SHINE, in Proceedings of the 39th International Free-Electron Laser Conference (JACOW, Geneva, Switzerland), pp. 199-202.

[20] M. L. Grünbein and G. N. Kovacs, Sample delivery for serial crystallography at free-electron lasers and synchrotrons, Acta Crystallogr., Sect. D: Struct. Biol. 75, 178 (2019).

[21] D. P. DePonte, U. Weierstall, K. Schmidt, J. Warner, D. Starodub, J. C. H. Spence, and R. B. Doak, Gas dynamic virtual nozzle for generation of microscopic droplet streams, J. Phys. D: Appl. Phys. 41, 195505 (2008).

[22] U. Weierstall, J. C. H. Spence, and R. B. Doak, Injector for scattering measurements on fully solvated biospecies, Rev. Sci. Instrum. 83, 035108 (2012).

[23] C. A. Stan, D. Milathianaki, H. Laksmono, R. G. Sierra, T. A. McQueen, M. Messerschmidt, G. J. Williams, J. E. Koglin, T. J. Lane, M. J. Hayes, S. A. H. Guillet, M. N. Liang, A. L. Aquila, P. R. Willmott, J. S. Robinson, K. L. Gumerlock, S. Botha, K. Nass, I. Schlichting, R. L. Shoeman et al., Liquid explosions induced by X-ray laser pulses, Nat. Phys. 12, 966 (2016).

[24] M. O. Wiedorn, S. Awel, A. J. Morgan, K. Ayyer, Y. Gevorkov, H. Fleckenstein, N. Roth, L. Adriano, R. Bean, K. R. Beyerlein, J. Chen, J. Coe, F. Francisco Cruz-Mazo, T. Ekeberg, R. Graceffa, M. Heymann, D. A. Horke, J. Knoška, V. Mariani, R. Nazari et al., Rapid sample delivery for megahertz serial crystallography at X-ray FELs, IUCrJ 5, 574 (2018).

[25] G. Blaj, M. N. Liang, A. L. Aquila, P. R. Willmott, J. E. Koglin, R. G. Sierra, J. S. Robinson, S. Boutet, and C. A. Stan, Generation of high-intensity ultrasound through shock propagation in liquid jets, Phys. Rev. Fluids 4, 043401 (2019).

[26] D. D. Dlott, Ultrafast spectroscopy of shock waves in molecular materials, Annu. Rev. Phys. Chem. 50, 251 (1999).

[27] M. L. Grünbein, J. Bielecki, A. Gorel, M. Stricker, R. Bean, M. Cammarata, K. Dörner, L. Fröhlich, L. Hartmann, S. Hauf, M. Hilpert, Y. Kim, M. Kloos, R. Letrun, M. Messerschmidt, G. Mills, G. Nass Kovacs, M. Ramilli, C. M. Roome, T. Sato et al., Megahertz data collection from protein microcrystals at an X-ray free-electron laser, Nat. Commun. 9, 3487 (2018).

[28] M. O. Wiedorn, D. Oberthür, R. Bean, R. Schubert, N. Werner, B. Abbey, M. Aepfelbacher, A. Adriano, A. Allahgholi, N. AlQudami, J. Andreasson, A. Aplin, S. Awel, K. Ayyer, S. Bajt, I. Barak, S. Bari, J. Bielecki, S. Botha, D. Boukhelef, W. Brehm et al., Megahertz serial crystallography, Nat. Commun. 9, 4025 (2018).

[29] O. Yefanov, D. Oberthür, R. Bean, M. O. Wiedorn, J. Knoska, G. Pena, S. Awel, L. Gumprecht, M. Domaracky, I. Sarrou, P. L. Xavier, M. Metz, S. Bajt, V. Mariani, Y. Gevorkov, T. A. White, A. Tolstikova, P. Villanueva-Perez, C. Seuring, S. Aplin et al., Evaluation of serial crystallographic structure determination within megahertz pulse trains, Struct. Dyn. 6, 064702 (2019).

[30] D. Ursescu, V. Aleksandrov, D. Matei, I. Dancus, M. D. de Almeida, and C. A. Stan, Generation of shock trains in free liquid jets with a nanosecond green laser, Phys. Rev. Fluids $\mathbf{5}$, 123402 (2020).

[31] F.-.J. Decker, R. Akre, A. Brachmann, Y. Ding, D. Dowell, P. J. Emma, A. Fisher, J. Frisch, S. Gilevich, P. Hering, Z. Huang, R. Iverson, H. Loos, M. Messerschmidt, H.-D. Nuhn,
D. Ratner, W. F. Schlotter, T. Smith, J. L. Turner, J. Welch et al., A demonstration of multi-bunch operation in the LCLS, in Proceedings of the 32nd Free-Electron Laser Conference (FEL2010) (JACOW, Geneva, Switzerland, 2010), pp. 467-470.

[32] F. J. Decker, K. L. F. Bane, W. Colocho, A. A. Lutman, and J. C. Sheppard, Recent developments and plans for two bunch operation with up to $1 \mu$ s separation at LCLS, Report SLACPUB-17128 (SLAC National Accelerator Laboratory, Menlo Park, CA, 2017).

[33] Y. W. Sun, D. L. Zhu, S. H. Song, F. J. Decker, M. Sutton, K. Ludwig, W. Roseker, G. Gruebel, S. Hruszkewycz, G. B. Stephenson, P. H. Fuoss, and A. Robert, Characterization of the LCLS "nanosecond two-bunch" mode for X-ray Speckle Visibility Spectroscopy experiments, in Advances in X-Ray Free-Electron Lasers Instrumentation IV, Proceedings of SPIE, Vol. 10237, edited by T. Tschentscher and L. Patthey (SPIE, Bellingham, WA, 2017), p. 102370N.

[34] M. N. Liang, G. J. Williams, M. Messerschmidt, M. M. Seibert, P. A. Montanez, M. Hayes, D. Milathianaki, A. Aquila, M. S. Hunter, J. E. Koglin, D. W. Schafer, S. Guillet, A. Busse, R. Bergan, W. Olson, K. Fox, N. Stewart, R. Curtis, A. A. Miahnahri, and S. Boutet, The coherent x-ray imaging instrument at the linac coherent light source, J. Synchrotron Radiat. 22, 514 (2015).

[35] K. R. Ferguson, M. Bucher, T. Gorkhover, S. Boutet, H. Fukuzawa, J. E. Koglin, Y. Kumagai, A. Lutman, A. Marinelli, M. Messerschmidt, K. Nagaya, J. Turner, K. Ueda, G. J. Williams, P. H. Bucksbaum, and C. Bostedt, Transient lattice contraction in the solid-to-plasma transition, Sci. Adv. 2, e1500837 (2016).

[36] K. Nass, A. Gorel, M. M. Abdullah, A. V. Martin, M. Kloos, A. Marinelli, A. Aquila, T. R. M. Barends, F. J. Decker, R. B. Doak, L. Foucar, E. Hartmann, M. Hilpert, M. S. Hunter, Z. Jurek, J. E. Koglin, A. Kozlov, A. A. Lutman, G. N. Kovacs, C. M. Roome et al., Structural dynamics in proteins induced by and probed with X-ray free-electron laser pulses, Nat. Commun. 11, 1814 (2020).

[37] D. Rich, D. L. Zhu, J. Turner, D. H. Zhang, B. Hill, and Y. P. Feng, The LCLS variable-energy hard X-ray single-shot spectrometer, J. Synchrotron Radiat. 23, 3 (2016).

[38] A. Katrusiak and Z. Dauter, Compressibility of lysozyme protein crystals by X-ray diffraction, Acta Crystallogr., Sect. D: Biol. Crystallogr. 52, 607 (1996).

[39] H. Yamada, T. Nagae, and N. Watanabe, High-pressure protein crystallography of hen egg-white lysozyme, Acta Crystallogr., Sect. D: Struct. Biol. 71, 742 (2015).

[40] S. Tait, E. T. White, and J. D. Litster, Mechanical characterization of protein crystals, Part. Part. Syst. Charact. 25, 266 (2008).

[41] H. Koizumi, H. Kawamoto, M. Tachibana, and K. Kojima, Effect of intracrystalline water on micro-Vickers hardness in tetragonal hen egg-white lysozyme single crystals, J. Phys. D: Appl. Phys. 41, 074019 (2008).

[42] L. Foucar, A. Barty, N. Coppola, R. Hartmann, P. Holl, U. Hoppe, S. Kassemeyer, N. Kimmel, J. Kupper, M. Scholz, S. Techert, T. A. White, L. Struder, and J. Ullrich, CASSCFEL-ASG software suite, Comput. Phys. Commun. 183, 2207 (2012).

[43] L. Foucar, CFEL-ASG software suite (CASS): usage for freeelectron laser experiments with biological focus, J. Appl. Crystallogr. 49, 1336 (2016). 
[44] See Supplemental Material at http://link.aps.org/supplemental/ 10.1103/PhysRevResearch.3.013046 which contains the crystallographic data statistics for the probe-equalized data sets and for the full data sets, and a discussion of Fig. 2 data.

[45] J. M. M. Pinkerton, A pulse method for the measurement of ultrasonic absorption in liquids: Results for water, Nature (London) 160, 128 (1947).

[46] C. A. Stan, K. Motomura, G. Blaj, Y. Kumagai, Y. W. Li, D. You, T. Ono, A. Kalita, T. Togashi, S. Owada, K. Tono, M. Yabashi, T. Katayama, and K. Ueda, The magnitude and waveform of shock waves induced by X-ray lasers in water, Appl. Sci. 10, 1497 (2020).

[47] A. Allahgholi, J. Becker, A. Delfs, R. Dinapoli, P. Goettlicher, D. Greiffenberg, B. Henrich, H. Hirsemann, M. Kuhn, R. Klanner, A. Klyuev, H. Krueger, S. Lange, T. Laurus, A. Marras, D. Mezza, A. Mozzanica, M. Niemann, J. Poehlsen, J. Schwandt et al., The Adaptive Gain Integrating Pixel Detector at the European XFEL, J. Synchrotron Radiat. 26, 74 (2019).

[48] W. J. Evans, C. S. Yoo, G. W. Lee, H. Cynn, M. J. Lipp, and K. Visbeck, Dynamic diamond anvil cell (dDAC): A novel device for studying the dynamic-pressure properties of materials, Rev. Sci. Instrum. 78, 073904 (2007).

[49] T. R. M. Barends, L. Foucar, S. Botha, R. B. Doak, R. L. Shoeman, K. Nass, J. E. Koglin, G. J. Williams, S. Boutet, M. Messerschmidt, and I. Schlichting, De novo protein crystal structure determination from X-ray free-electron laser data, Nature (London) 505, 244 (2014).
[50] L. Lomb, J. Steinbrener, S. Bari, D. Beisel, D. Berndt, C. Kieser, M. Lukat, N. Neef, and R. L. Shoeman, An anti-settling sample delivery instrument for serial femtosecond crystallography, J. Appl. Crystallogr. 45, 674 (2012).

[51] M. L. Grünbein, R. L. Shoeman, and R. B. Doak, Velocimetry of fast microscopic liquid jets by nanosecond dual-pulse laser illumination for megahertz X-ray free-electron lasers, Opt. Express 26, 7190 (2018).

[52] T. A. White, R. A. Kirian, A. V. Martin, A. Aquila, K. Nass, A. Barty, and H. N. Chapman, CrystFEL: a software suite for snapshot serial crystallography, J. Appl. Crystallogr. 45, 335 (2012).

[53] G. N. Murshudov, A. A. Vagin, and E. J. Dodson, Refinement of macromolecular structures by the maximum-likelihood method, Acta Crystallogr., Sect. D: Struct. Biol. 53, 240 (1997).

[54] P. Emsley, B. Lohkamp, W. G. Scott, and K. Cowtan, Features and development of Coot, Acta Crystallogr., Sect. D: Biol. Crystallogr. 66, 486 (2010).

[55] R. A. Kirian, X. Y. Wang, U. Weierstall, K. E. Schmidt, J. C. H. Spence, M. Hunter, P. Fromme, T. White, H. N. Chapman, and J. Holton, Femtosecond protein nanocrystallography-data analysis methods, Opt. Express 18, 5713 (2010).

[56] S. Bailey, The CCP4 suite: Programs for protein crystallography, Acta Crystallogr., Sect. D: Biol. Crystallogr. 50, 760 (1994).

[57] P. Emsley and K. Cowtan, Coot: model-building tools for molecular graphics, Acta Crystallogr., Sect. D: Struct. Biol. 60, 2126 (2004). 